\title{
The Drell-Yan process with pions and polarized nucleons
}

\author{
S. Bastami, ${ }^{a}$ L. Gamberg, ${ }^{b}$ B. Parsamyan, ${ }^{c, d}$ B. Pasquini, ${ }^{e, f}$ A. Prokudin ${ }^{b, g}$ and \\ P. Schweitzer ${ }^{a}$ \\ ${ }^{a}$ Department of Physics, University of Connecticut, \\ Storrs, CT 06269, U.S.A. \\ ${ }^{b}$ Division of Science, Penn State Berks, \\ Reading, PA 19610, U.S.A. \\ ${ }^{c}$ Dipartimento di Fisica, Università degli Studi di Torino, \\ Torino, Italy \\ ${ }^{d}$ Istituto Nazionale di Fisica Nucleare, Sezione di Torino, \\ Torino, Italy \\ e Dipartimento di Fisica, Università degli Studi di Pavia, \\ Pavia, Italy \\ ${ }^{f}$ Istituto Nazionale di Fisica Nucleare, Sezione di Pavia, \\ Pavia, Italy \\ ${ }^{g}$ Thomas Jefferson National Accelerator Facility, \\ Newport News, VA 23606, U.S.A. \\ E-mail: saman.bastami@uconn.edu, lpg10@psu.edu, bakur@cern.ch, \\ barbara.pasquini@unipv.it, prokudin@jlab.org, \\ peter.schweitzer@uconn.edu
}

AbStRACT: The Drell-Yan process provides important information on the internal structure of hadrons including transverse momentum dependent parton distribution functions (TMDs). In this work we present calculations for all leading twist structure functions describing the pion induced Drell-Yan process. The non-perturbative input for the TMDs is taken from the light-front constituent quark model, the spectator model, and available parametrizations of TMDs extracted from the experimental data. TMD evolution is implemented at Next-to-Leading Logarithmic precision for the first time for all asymmetries. Our results are compatible with the first experimental information, help to interpret the data from ongoing experiments, and will allow one to quantitatively assess the models in future when more precise data will become available.

KEYWorDs: Deep Inelastic Scattering (Phenomenology)

ARXIV EPRINT: 2005.14322 


\section{Contents}

1 Introduction 1

2 Drell-Yan process with pions and polarized protons 3

2.1 Structure functions 4

2.2 QCD evolution of Drell-Yan structure functions 5

2.3 Input for TMDs and choice of the initial scale $Q_{0} \quad 8$

$\begin{array}{lll}2.4 & \text { TMDs extracted from experimental data } & 10\end{array}$

2.5 TMDs from models 11

3 Results and observations $\quad 14$

3.1 The COMPASS Drell-Yan experiment 14

$\begin{array}{ll}3.2 & \text { The approaches for numerical estimates } \\ 3.3 & 15\end{array}$

$\begin{array}{lll}3.3 & \text { Discussion of the results and comparison to available data } & 15\end{array}$

$\begin{array}{llr}4 \text { Conclusions } & 20\end{array}$

\section{Introduction}

The Drell-Yan (DY) process with pions and nucleons provides important information on the structure of pion and nucleon. The DY differential cross section in the region of low transverse momentum, $q_{T}$, of the produced lepton anti-lepton pair is subject to the transverse momentum dependent factorization [1]. The corresponding transverse momentum dependent parton distribution functions (TMDs) [2] in the description of DY at low $q_{T}$ provide essential information on correlations between transverse parton momenta and parton or nucleon spin, and describe the three-dimensional structure of hadrons. Early theoretical studies of TMDs in hadron production in proton-proton processes [3-5] were followed by systematic investigations in semi-inclusive deep-inelastic scattering (SIDIS) [6-9] and DY [10-12] (also fragmentation functions [13] enter the description of SIDIS). The basis for these descriptions are QCD factorization theorems [1, 2, 14-22].

One of the challenges when interpreting pion-induced DY data is the limited knowledge of the pion structure. At twist-2 the process is described by the proton TMDs: unpolarized distribution $f_{1, p}^{a}$, transversity distribution $h_{1, p}^{a}$, Sivers distribution function $f_{1 T, p}^{\perp a}$, Boer-Mulders distribution $h_{1, p}^{\perp a}$, Kotzinian-Mulders distribution $h_{1 L, p}^{\perp a}$, and "pretzelosity" distribution $h_{1 T, p}^{\perp a}$, and pion TMDs: unpolarized distribution $f_{1, \pi}^{a}$, Boer-Mulders distribution $h_{1, \pi}^{\perp a}$.

On the proton side, for $f_{1, p}^{a}$ both collinear and TMD distributions are wellknown [23-33]. Based on global QCD analyses of data, parametrizations are available also for $f_{1 T, p}^{\perp a}, h_{1, p}^{a}, h_{1, p}^{\perp a}, h_{1 T, p}^{\perp a}$ [34-38]. Only $h_{1 L, p}^{\perp a}$ has not yet been extracted, though it can 
be described based on $h_{1, p}^{a}$ in the so-called Wandzura-Wilczek- (WW-)type approximation which is compatible with available data [39]. On the pion side the situation is different. While extractions of $f_{1, \pi}^{a}$ exist [40-45], no results on $h_{1, \pi}^{\perp a}$ are available. This constitutes a "bottleneck" if one would like to describe the pion-induced DY data, e.g. COMPASS results [46], based solely on phenomenological extractions since $h_{1, \pi}^{\perp a}$ is relevant for the majority of observables in the pion-induced polarized DY process at leading twist. In this situation we will resort to model studies of the pion Boer-Mulders function $h_{1, \pi}^{\perp a}$.

An important goal of theoretical studies in models is to describe hadron structure at a low initial scale $\mu_{0}<1 \mathrm{GeV}$ in terms of effective constituent quark degrees of freedom. This approach has been successful in describing various hadronic properties in terms of "valence-quark degrees of freedom." The underlying idea is that at a low hadronic scale $\mu_{0}$, for example the properties of the nucleon can be modelled in terms of wave functions of valence $u$ and $d$ quarks, and similarly the properties of the $\pi^{-}$in terms of the wave functions of valence $\bar{u}$ and $d$ quarks. It is an interesting task in itself to apply such a framework to the description of hadronic properties like TMDs. This has been done in a variety of complementary approaches including chiral quark models [47] and generalizations [48], spectator models (SPMs) [49-53], light-front constituent quark model (LFCQM) [54-61] or bag models [62-66]. Phenomenological studies in the LFCQM showed that within a model accuracy of 20-30\% a good description of SIDIS and unpolarized DY data can be obtained [57-59].

The goal of the present work is to study the spin and azimuthal asymmetries in the DY process with pions and polarized nucleons, and to present calculations for all twist-2 asymmetries. We use available phenomenological extractions of TMDs and calculations from two well-established constituent-quark-models (CQM), the LFCQM and the SPM. Other studies in models, perturbative QCD and lattice QCD of the pion-induced DY or relevant TMDs have been reported [67-73].

Several features distinguish our work from other studies. First, we use two CQM frameworks with diverse descriptions of the pion and nucleon structure. Second, we describe all leading-twist observables in pion-induced polarized DY entirely in the models. Third, we supplement our studies with "hybrid calculations", where we use as much as possible information from phenomenological analyses, and only the Boer-Mulders function $h_{1, \pi}^{\perp a}$ is taken from models. Overall, we present up to four different calculations for each observable. This allows us to critically assess model dependence, and uncertainties in our approach. Where available the results are compared to the COMPASS DY data [46].

One key aspect in our study is the evolution of model results from the low hadronic scales to experimentally relevant scales. For that (i) knowledge of the low initial scale, and (ii) applicability of evolution equations at low scales are crucial. Both requirements are fulfilled in the case of parton distribution functions which depend on one scale only, the renormalization scale $\mu$. First, the value of the initial quark model scale $\mu_{0}$ can be consistently determined by evolving the fraction of nucleon momentum carried by valence quarks, $M_{2}^{\text {val }}(\mu)=\sum_{a} \int d x x\left(f_{1}^{q}-f_{1}^{\bar{q}}\right)(x, \mu)$, known from parametrizations, using DGLAP evolution down to that scale $\mu_{0}$ at which valence quarks carry the entire nucleon momentum, i.e. $M_{2}^{\text {val }}\left(\mu_{0}\right)=1$ [74]. Numerically it is $\mu_{0} \sim 0.5 \mathrm{GeV}$. Second, works by the GRV and GRS 
groups on parametrizations of nucleon and pion unpolarized parton distribution functions show remarkable perturbative stability between LO and NLO fits indicating applicability of DGLAP evolution down to initial scales as low as $\mu_{0}^{2}=0.26 \mathrm{GeV}^{2}[23-25,40,42]$.

TMDs depend not only on the renormalization scale $\mu$ but also on the rapidity scale $\zeta$ [2]. The theoretical and phenomenological understanding of TMDs witnessed an incredible rate of developments in the recent years including NNLO and NNNLO calculations of the evolution kernel of unpolarized TMDs [75-83], NLO calculations for the quark helicity distribution [84], NLO [84] and NNLO [85] calculations for transversity and pretzelosity, and NLO calculations for the Sivers function [86-90]. Recently also the first non-trivial expression for the small- $b$ expansion of the pretzelosity distribution was derived [91]. However, in the context of quark model applications we face two challenges. First, no rigorous (analog to the $\mu_{0}$-determination) criterion exists to fix the value of the initial rapidity scale $\zeta_{0}$ of quark models, though an educated guess may be $\zeta_{0} \sim \mu_{0}^{2}$. Secondly, in the case of Collins-Soper-Sterman (CSS) or TMD evolution [1, 2], no expertise is available analogous to the GRV/GRS applications of DGLAP evolution starting from low hadronic scales.

In this situation in previous quark model studies, TMD evolution effects were often estimated approximately [57-59] based on an heuristic Gaussian Ansatz for transverse parton momenta with energy dependent Gaussian widths. While providing a useful description of data on many processes including pion-induced Drell-Yan [92], it is important to improve the simple Gaussian treatment in view of the recent progress in the TMD theory [75-91]. We will therefore use TMD evolution [2] at Next-to-Leading Logarithmic (NLL) precision to describe the transverse momentum dependence of the Drell-Yan process. At present, application of TMD evolution at the low quark model scales below $1 \mathrm{GeV}$ is not known. Therefore, we shall proceed in two steps. We will evolve weighted transverse moments of TMDs from the low initial scale $\mu_{0}^{2}$ to a scale of $Q_{0}^{2}=2.4 \mathrm{GeV}^{2}$ where phenomenological information on transverse momentum dependence is available from TMD fits [32, 33, 93-96] of polarized and unpolarized SIDIS, DY and weak boson productions data. Then we use NLL TMD evolution to evolve to the scales relevant in the COMPASS Drell-Yan measurements, i.e., $\left\langle Q^{2}\right\rangle=28 \mathrm{GeV}^{2}$. In this way we will be able to test the $x$-dependencies of the model TMDs while the $q_{T}$-dependencies of the DY observables are described on the basis of TMD fits.

For completeness we remark that the importance of TMD evolution for the description of pion-induced DY and the recent COMPASS data was also studied in refs. [73, 97-101].

Our results serve several purposes. They help to interpret in their full complexity the first COMPASS data [46] on the pion-induced polarized DY process, and in this way deepen the understanding of the QCD description of deep-inelastic processes in terms of TMDs. They also provide quantitative tests of the application of CQMs to the description of pion and nucleon structure.

\section{Drell-Yan process with pions and polarized protons}

In this section we briefly review the DY formalism, and provide the description of the DY structure functions in our approach. 


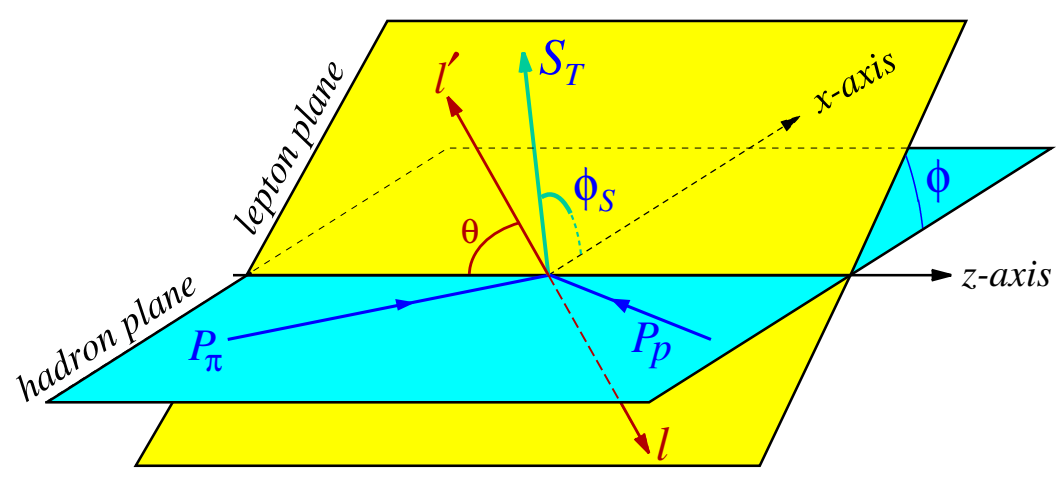

Figure 1. The DY process in the Collins-Soper frame where the pion and the proton come in with different momenta $P_{\pi}, P_{p}$, but each carries the same transverse momentum $\frac{1}{2} \boldsymbol{q}_{T}$, and the produced lepton pair is at rest. The angle $\phi$ describes the inclination of the leptonic frame with respect to the hadronic plane, and $\phi_{S}$ is the azimuthal angle of the transverse-spin vector of the proton.

\subsection{Structure functions}

In the tree-level description a dilepton $l, l^{\prime}$ is produced from the annihilation of a quark and antiquark carrying the fractions $x_{\pi}, x_{p}$ of the longitudinal momenta of respectively the pion and the proton. The process is shown in the Collins-Soper frame in figure 1. In the case of pions colliding with polarized protons the DY cross section is described in terms of six structure functions [12],

$$
\begin{aligned}
F_{U U}^{1} & =\mathcal{C}\left[f_{1, \pi}^{\bar{a}} f_{1, p}^{a}\right], \\
F_{U U}^{\cos 2 \phi} & =\mathcal{C}\left[\frac{2\left(\hat{\boldsymbol{h}} \cdot \overrightarrow{\boldsymbol{k}}_{T \pi}\right)\left(\hat{\boldsymbol{h}} \cdot \overrightarrow{\boldsymbol{k}}_{T p}\right)-\overrightarrow{\boldsymbol{k}}_{T \pi} \cdot \overrightarrow{\boldsymbol{k}}_{T p}}{M_{\pi} M_{p}} h_{1, \pi}^{\perp \bar{a}} h_{1, p}^{\perp a}\right], \\
F_{U L}^{\sin 2 \phi} & =-\mathcal{C}\left[\frac{2\left(\hat{\boldsymbol{h}} \cdot \overrightarrow{\boldsymbol{k}}_{T \pi}\right)\left(\hat{\boldsymbol{h}} \cdot \overrightarrow{\boldsymbol{k}}_{T p}\right)-\overrightarrow{\boldsymbol{k}}_{T \pi} \cdot \overrightarrow{\boldsymbol{k}}_{T p}}{M_{\pi} M_{p}} h_{1, \pi}^{\perp \bar{a}} h_{1 L, p}^{\perp a}\right], \\
F_{U T}^{\sin \phi_{S}} & =\mathcal{C}\left[\frac{\hat{\boldsymbol{h}} \cdot \overrightarrow{\boldsymbol{k}}_{T p}}{M_{p}} f_{1, \pi}^{\bar{a}} f_{1 T, p}^{\perp a}\right], \\
F_{U T}^{\sin \left(2 \phi-\phi_{S}\right)} & =-\mathcal{C}\left[\frac{\hat{\boldsymbol{h}} \cdot \overrightarrow{\boldsymbol{k}}_{T \pi}}{M_{\pi}} h_{1, \pi}^{\perp \bar{a}} h_{1, p}^{a}\right], \\
F_{U T}^{\sin \left(2 \phi+\phi_{S}\right)} & =-\mathcal{C}\left[\frac{2\left(\hat{\boldsymbol{h}} \cdot \overrightarrow{\boldsymbol{k}}_{T p}\right)\left[2\left(\hat{\boldsymbol{h}} \cdot \overrightarrow{\boldsymbol{k}}_{T \pi}\right)\left(\hat{\boldsymbol{h}} \cdot \overrightarrow{\boldsymbol{k}}_{T p}\right)-\overrightarrow{\boldsymbol{k}}_{T \pi} \cdot \overrightarrow{\boldsymbol{k}}_{T p}\right]-\overrightarrow{\boldsymbol{k}}_{T p}^{2}\left(\hat{\boldsymbol{h}} \cdot \overrightarrow{\boldsymbol{k}}_{T \pi}\right)}{2 M_{\pi} M_{p}^{2}} h_{1, \pi}^{\perp \bar{a}} h_{1 T, p}^{\perp a}\right] .
\end{aligned}
$$

The subscripts indicate the hadron polarization which can be unpolarized $U$ (pions, protons), longitudinally $L$, or transversely $T$ polarized (protons). The azimuthal angles $\phi, \phi_{S}$ are defined in figure 1 , where the unit vector $\hat{\boldsymbol{h}}=\boldsymbol{q}_{T} / q_{T}$ points along the $x$-axis. Notice that in the Collins-Soper frame the dilepton is at rest, and each incoming hadron carries the transverse momentum $\boldsymbol{q}_{T} / 2$, see figure 1 . The convolution integrals in eq. (2.1) are 
defined as [12]

$$
\mathcal{C}\left[\omega f_{\pi}^{\bar{a}} f_{p}^{a}\right]=\frac{1}{N_{c}} \sum_{a} e_{a}^{2} \int d^{2} \boldsymbol{k}_{T \pi} d^{2} \boldsymbol{k}_{T p} \delta^{(2)}\left(\boldsymbol{q}_{T}-\boldsymbol{k}_{T \pi}-\boldsymbol{k}_{T p}\right) \omega f_{\pi}^{\bar{a}}\left(x_{\pi}, \boldsymbol{k}_{T \pi}^{2}\right) f_{p}^{a}\left(x_{p}, \boldsymbol{k}_{T p}^{2}\right),
$$

where $\omega$, which is a function of the transverse momenta $\boldsymbol{k}_{T \pi}, \boldsymbol{k}_{T p}$ and $\boldsymbol{q}_{T}$, projects out the corresponding azimuthal angular dependence. The sum over $a=u, \bar{u}, d, \bar{d}, \ldots$ includes the active flavors.

This partonic interpretation of DY is based on a TMD factorization [1,2] and applies to the region $q_{T} \ll Q$. The TMDs depend on renormalization and rapidity scales which are not indicated for brevity in (2.1) and (2.2) and will be discussed in section 2.2. The focus of our work is on asymmetries of the kind

$$
A_{X Y}^{\text {weight }}\left(x_{\pi}, x_{p}, q_{T}, Q^{2}\right)=\frac{F_{X Y}^{\text {weight }}\left(x_{\pi}, x_{p}, q_{T}, Q^{2}\right)}{F_{U U}^{1}\left(x_{\pi}, x_{p}, q_{T}, Q^{2}\right)},
$$

where various types of higher order corrections tend to largely cancel out [102-108].

The $Q^{2}$ dependence of the structure functions and asymmetries will often not be explicitly indicated for brevity. In the following we will display results for the asymmetries as functions of one of the variables $x_{\pi}, x_{p}, q_{T}$. It is then understood that the structure functions are integrated over the other variables within the acceptance of the experiment, keeping in mind that $x_{\pi}, x_{p}$ are connected to each other by $x_{\pi} x_{p}=Q^{2} / s$, where $s$ is the center of mass energy squared.

\subsection{QCD evolution of Drell-Yan structure functions}

The basis for the evolution are TMD factorization theorems [1, 2, 14-22, 109] which constrain the operator definition and define the QCD evolution of TMDs. Here we will adopt the CSS framework and use the TMD evolution formalism starting from a fixed scale $Q_{0}$ [21] in the structure functions from eqs. (2.1).

The evolution of TMDs is a double-scale problem, and can be implemented in momentum space or impact-parameter space with examples for both approaches in the literature $[19,32,33,110-112]$. In our work we choose to implement the TMD evolution in the impact-parameter space with $\boldsymbol{b}_{T}$ the Fourier-conjugate variable to $\boldsymbol{k}_{T h}$ where in$\operatorname{dex} h=\pi$ or $p$ refers to pion or nucleon. The TMDs in the impact-parameter space are generically given by $\tilde{f}\left(x_{h}, b_{T}, \mu, \zeta\right)$ where $\mu \sim Q$ is the "standard" renormalization scale for ultraviolet logarithms, and $\zeta \sim Q^{2}$ is the rapidity renormalization scale. In principle one can solve TMD evolution equations starting from some initial scale $Q_{0}$ without employing operator product expansion at low $\boldsymbol{b}_{T}$, ref. [21]. The TMD at this initial scale is then $f\left(x_{h}, b_{T}, Q_{0}, Q_{0}^{2}\right)$. In this formulation the unpolarized structure function is similar to parton model result and is expressed as [21]

$$
\begin{aligned}
F_{U U}^{1}\left(x_{\pi}, x_{p}, q_{T}, Q^{2}\right)= & \frac{1}{N_{c}} \sum_{a} e_{a}^{2} \mathcal{H}^{(D Y)}\left(Q, \mu_{Q}\right) \int \frac{b_{T} d b_{T}}{2 \pi} J_{0}\left(q_{T} b_{T}\right) \\
& \times f_{1, \pi}^{\bar{a}}\left(x_{\pi}, b_{T}, Q_{0}, Q_{0}^{2}\right) \tilde{f}_{1, p}^{a}\left(x_{p}, b_{T}, Q_{0}, Q_{0}^{2}\right) e^{-S\left(b_{T}, Q_{0}, Q, \mu_{Q}\right)},
\end{aligned}
$$


where the factor $S\left(b_{T}, Q_{0}, \mu_{Q}\right)$ contains important effects of gluon radiation with $S\left(b_{T}, Q_{0}, Q_{0}\right)=0$ by construction [21]. The hard factor $\mathcal{H}\left(Q, \mu_{Q}\right)$ is [113]

$$
\mathcal{H}^{(D Y)}\left(Q, \mu_{Q}\right)=1+\frac{\alpha_{s}\left(\mu_{Q}\right)}{2 \pi} C_{F}\left(3 \ln \left(\frac{Q^{2}}{\mu_{Q}^{2}}\right)-\ln ^{2}\left(\frac{Q^{2}}{\mu_{Q}^{2}}\right)+\frac{7 \pi^{2}}{6}-8\right)+\mathcal{O}\left(\alpha_{s}^{2}\right),
$$

where $C_{F}=4 / 3$ and $\alpha_{s}$ is the strong coupling constant.

One can parametrize TMDs at initial scale $Q_{0}$ as

$$
\begin{aligned}
& \tilde{f}_{1, p}^{a}\left(x_{p}, b_{T}, Q_{0}, Q_{0}^{2}\right)=f_{1, p}^{a}\left(x_{p}, Q_{0}\right) e^{-\frac{1}{4} b_{T}^{2}\left\langle k_{T p}^{2}\right\rangle_{1, p}}, \\
& \tilde{f}_{1, \pi}^{a}\left(x_{\pi}, b_{T}, Q_{0}, Q_{0}^{2}\right)=f_{1, \pi}^{a}\left(x_{\pi}, Q_{0}\right) e^{-\frac{1}{4} b_{T}^{2}\left\langle k_{T \pi}^{2}\right\rangle_{f_{1, \pi}}},
\end{aligned}
$$

where $x$-dependent functions correspond to collinear distributions and the exponential factors are "primordial shapes" of TMDs at the initial scale. This particular dependence is often used in phenomenology [92, 114], corresponds to the Gaussian Ansatz and is supported in models $[58,59,66,115,116]$. The average widths of TMDs may be flavor- and $x$-dependent and will be taken from phenomenological parametrizations at $Q_{0}^{2}$.

Based on the $b_{T}$ space formalism given in ref. [117] we write down the rest of the twist-2 structure functions. We use the convenient notation from ref. [118],

$$
\begin{aligned}
\mathcal{B}_{n}\left[\tilde{f}_{\pi} \tilde{f}_{p}\right] \equiv & \frac{1}{N_{c}} \sum_{a} e_{a}^{2} \mathcal{H}^{(D Y)}\left(Q, \mu_{Q}\right) \int_{0}^{\infty} \frac{d b_{T} b_{T}}{2 \pi} b_{T}^{n} J_{n}\left(q_{T} b_{T}\right) \\
& \times \tilde{f}_{\pi}^{\bar{a}}\left(x_{\pi}, b_{T}, Q_{0}, Q_{0}^{2}\right) \tilde{f}_{p}^{a}\left(x_{p}, b_{T}, Q_{0}, Q_{0}^{2}\right) e^{-S\left(b_{T}, Q_{0}, Q, \mu_{Q}\right)}
\end{aligned}
$$

which leads to the following expressions for the twist-2 structure functions,

$$
\begin{aligned}
F_{U U}^{1}\left(x_{\pi}, x_{p}, q_{T}, Q^{2}\right) & =\mathcal{B}_{0}\left[\tilde{f}_{1, \pi} \tilde{f}_{1, p}\right], \\
F_{U U}^{\cos 2 \phi}\left(x_{\pi}, x_{p}, q_{T}, Q^{2}\right) & =M_{\pi} M_{p} \mathcal{B}_{2}\left[\tilde{h}_{1, \pi}^{\perp(1)} \tilde{h}_{1, p}^{\perp(1)}\right], \\
F_{U L}^{\sin 2 \phi}\left(x_{\pi}, x_{p}, q_{T}, Q^{2}\right) & =-M_{\pi} M_{p} \mathcal{B}_{2}\left[\tilde{h}_{1, \pi}^{\perp(1)} \tilde{h}_{1 L, p}^{\perp(1)}\right], \\
F_{U T}^{\sin \phi_{S}}\left(x_{\pi}, x_{p}, q_{T}, Q^{2}\right) & =M_{p} \mathcal{B}_{1}\left[\tilde{f}_{1, \pi} \tilde{f}_{1 T, p}^{\perp(1)}\right], \\
F_{U T}^{\sin \left(2 \phi-\phi_{S}\right)}\left(x_{\pi}, x_{p}, q_{T}, Q^{2}\right) & =-M_{\pi} \mathcal{B}_{1}\left[\tilde{h}_{1, \pi}^{\perp(1)} \tilde{h}_{1, p}\right], \\
F_{U T}^{\sin \left(2 \phi+\phi_{S}\right)}\left(x_{\pi}, x_{p}, q_{T}, Q^{2}\right) & =-\frac{M_{\pi} M_{p}^{2}}{4} \mathcal{B}_{3}\left[\tilde{h}_{1, \pi}^{\perp(1)} \tilde{h}_{1 T, p}^{\perp(2)}\right],
\end{aligned}
$$

where the $b_{T}$ space TMD moments [117] are

$$
\tilde{f}^{(n)}\left(x_{h}, b_{T}, Q, Q^{2}\right)=(-1)^{n} n !\left(\frac{2}{M_{h}^{2}} \frac{\partial}{\partial b_{T}^{2}}\right)^{n} \tilde{f}\left(x_{h}, b_{T}, Q, Q^{2}\right) .
$$

These moments have the important feature,

$$
\lim _{b_{T} \rightarrow 0} \tilde{f}^{(n)}\left(x_{h}, b_{T}, Q, Q^{2}\right)=f^{(n)}\left(x_{h}, Q\right),
$$

where $f^{(n)}$ are conventional transverse moments of TMDs [5] defined as

$$
f^{(n)}\left(x_{h}, Q\right)=\int \mathrm{d}^{2} \boldsymbol{k}_{T h}\left(\frac{\boldsymbol{k}_{T h}^{2}}{2 M_{h}^{2}}\right)^{n} f\left(x_{h}, \boldsymbol{k}_{T h}^{2}, Q, Q^{2}\right),
$$


and $h=\pi, p$ corresponds to pion and proton TMDs, respectively. The evolution factor $S\left(b_{T}, Q_{0}, \mu_{Q}\right)$ in eq. (2.8), which results from solving the CSS evolution equation and the renormalization group equations for the rapidity dependence of the TMDs and for the soft factor $[2,19]$, is given by

$$
S\left(b_{T}, Q_{0}, Q, \mu_{Q}\right)=-\tilde{K}\left(b_{T}, Q_{0}\right) \ln \frac{Q^{2}}{Q_{0}^{2}}+\int_{Q_{0}}^{\mu_{Q}} \frac{d \bar{\mu}}{\bar{\mu}}\left[\gamma_{K}\left(\alpha_{s}(\bar{\mu})\right) \ln \frac{\mu_{Q}^{2}}{\bar{\mu}^{2}}-2 \gamma_{i}\left(\alpha_{s}(\bar{\mu}) ; 1\right)\right],
$$

where $\tilde{K}$ is the Collins-Soper evolution kernel, and the anomalous dimensions are $\gamma_{i}\left(\alpha_{s}(\bar{\mu}) ; 1\right)$ and $\gamma_{K}\left(\alpha_{s}(\bar{\mu})\right)[21]$.

Since the integral in eq. (2.8) extends over all $b_{T}$, one cannot avoid using $\tilde{K}$ in the CSS evolution factor (2.18) in the non-perturbative large $b_{T}$ region. In order to combine the perturbative and non-perturbative regions, we use the $b_{*}$ prescription [1], namely,

$$
b_{*}=\frac{b_{T}}{\sqrt{1+b_{T}^{2} / b_{\max }^{2}}},
$$

which introduces a smooth upper cutoff $b_{\max }$ in the transverse distance.

Then, the perturbative part of $\tilde{K}$ is defined by replacing $b_{T}$ by $b_{*}$ and the nonperturbative part is defined by the difference $\tilde{K}\left(b_{*}, \mu\right)-\tilde{K}\left(b_{T}, \mu\right)=g_{K}\left(b_{T} ; b_{\max }\right)$ [21]. Furthermore to combine the perturbative and non-perturbative regions using the fixed scale evolution, it is optimal to use the renormalization group running scheme for $\tilde{K}$ in eq. (2.18), evolved from the fixed scale $Q_{0}$, i.e.

$$
\tilde{K}\left(b_{T}, Q_{0}\right)=\tilde{K}\left(b_{*}, \mu_{b_{*}}\right)-\int_{\mu_{b_{*}}}^{Q_{0}} \frac{d \bar{\mu}}{\bar{\mu}} \gamma_{K}\left(\alpha_{s}(\bar{\mu})\right)-g_{K}\left(b_{T} ; b_{\max }\right)
$$

where $\mu_{b_{*}}$ is now chosen to become a hard scale,

$$
\mu_{b_{*}} \equiv \frac{C_{1}}{b_{*}}
$$

Now eq. (2.18) reads [21]

$$
\begin{aligned}
S\left(b_{T}, b_{*}, Q_{0}, Q, \mu_{Q}\right)= & \left(g_{K}\left(b_{T} ; b_{\max }\right)-\tilde{K}\left(b_{*} ; \mu_{b_{*}}\right)+\int_{\mu_{b_{*}}}^{Q_{0}} \frac{d \bar{\mu}}{\bar{\mu}} \gamma_{K}\left(\alpha_{s}(\bar{\mu})\right)\right) \ln \frac{Q^{2}}{Q_{0}^{2}} \\
& +\int_{Q_{0}}^{\mu_{Q}} \frac{d \bar{\mu}}{\bar{\mu}}\left[\gamma_{K}\left(\alpha_{s}(\bar{\mu})\right) \ln \frac{\mu_{Q}^{2}}{\bar{\mu}^{2}}-2 \gamma_{i}\left(\alpha_{s}(\bar{\mu}) ; 1\right)\right] .
\end{aligned}
$$

The anomalous dimensions can be expanded as perturbative series, $\gamma_{i}=\sum_{n=1}^{\infty} \gamma_{i}^{(n)}\left(\alpha_{s} / \pi\right)^{n}$, and $\gamma_{K}=\sum_{n=1}^{\infty} \gamma_{K}^{(n)}\left(\alpha_{s} / \pi\right)^{n}$. In our calculations we employ them to NLL accuracy: $\gamma_{K}^{(1)}$, $\gamma_{K}^{(2)}$ and $\gamma_{i}^{(1)}$. They are spin-independent [1, 19, 21, 29, 119-123], and given by

$$
\gamma_{K}^{(1)}=2 C_{F}, \quad \gamma_{K}^{(2)}=C_{F}\left[C_{A}\left(\frac{67}{18}-\frac{\pi^{2}}{6}\right)-\frac{10}{9} T_{F} n_{f}\right], \quad \gamma_{i}^{(1)}=\frac{3}{2} C_{F},
$$


where $C_{F}=4 / 3, C_{A}=3, T_{F}=1 / 2$ and $n_{f}$ is the number of active flavors. The NLL two-loop contribution for $\tilde{K}[113,124]$, valid at small values of $b_{T}$, is

$$
\begin{aligned}
\tilde{K}\left(b_{*} ; \mu_{b_{*}}\right)= & -2 C_{F} \frac{\alpha_{s}\left(\mu_{b_{*}}\right)}{\pi} \ln \left(\frac{b_{*} \mu_{b_{*}}}{2 e^{-\gamma_{E}}}\right)+\frac{C_{F}}{2}\left(\frac{\alpha_{s}\left(\mu_{b_{*}}\right)}{\pi}\right)^{2}\left[\left(\frac{2}{3} n_{f}-\frac{11}{3} C_{A}\right) \ln ^{2}\left(\frac{b_{*} \mu_{b_{*}}}{2 e^{-\gamma_{E}}}\right)\right. \\
& \left.+\left(-\frac{67}{9} C_{A}+\frac{\pi^{2}}{3} C_{A}+\frac{10}{9} n_{f}\right) \ln \left(\frac{b_{*} \mu_{b_{*}}}{2 e^{-\gamma_{E}}}\right)+\left(\frac{7}{2} \zeta_{3}-\frac{101}{27}\right) C_{A}+\frac{28}{27} T_{F} n_{f}\right],
\end{aligned}
$$

so that for $C_{1}=2 e^{-\gamma_{E}}$, one finds

$$
\tilde{K}\left(b_{*} ; \mu_{b_{*}}\right)=\frac{C_{F}}{2}\left(\frac{\alpha_{s}\left(\mu_{b_{*}}\right)}{\pi}\right)^{2}\left[\left(\frac{7}{2} \zeta_{3}-\frac{101}{27}\right) C_{A}+\frac{28}{27} T_{F} n_{f}\right] .
$$

We will numerically calculate the integral in eq. (2.18) using the two-loop result for the strong coupling constant, tuned to the world average [125] $\alpha_{s}\left(M_{Z}\right)=0.118$ as in the CTEQ analysis [126].

Furthermore, we adopt the functional form of $g_{K}\left(b_{T} ; b_{\max }\right)$ given by Collins and Rogers [21],

$$
g_{K}\left(b_{T}, b_{\max }\right)=g_{0}\left(b_{\max }\right)\left(1-\exp \left[-\frac{C_{F} \alpha_{s}\left(\mu_{b_{*}}\right) b_{T}^{2}}{\pi g_{0}\left(b_{\max }\right) b_{\max }^{2}}\right]\right)
$$

which interpolates smoothly between the small and large- $b_{T}$ regions, where at small $b_{T}$ it approximates a power series in $b_{T}^{2}$, while at large $b_{T}$ the resulting value of $\tilde{K}$ goes to a constant [21]. We choose $g_{0}\left(b_{\max }\right)=0.84$ and $b_{\max }=1 \mathrm{GeV}^{-1}$ to match the nonperturbative behavior of $g_{K}$ used in refs. [94, 95] to describe the polarized SIDIS data and in ref. [93] to describe unpolarized SIDIS, DY and weak boson production data. The scale $\mu_{Q}$ is usually chosen such that $\mu_{Q}=C_{2} Q$. In the following we will use $C_{2}=1$ and $C_{1}=2 e^{-\gamma_{E}}$. These choices allow one to optimize the accuracy of the perturbative expansion calculations in eqs. (2.5) and (2.24) [113].

\subsection{Input for TMDs and choice of the initial scale $Q_{0}$}

We will utilize the following parametrizations [39] for TMDs at the initial scale $Q_{0}$ :

$$
\begin{aligned}
& f_{h}^{a}\left(x_{h}, \boldsymbol{k}_{T h}, Q_{0}, Q_{0}^{2}\right)=f_{h}^{a}\left(x_{h}, Q_{0}\right) \frac{e^{-\boldsymbol{k}_{T h}^{2} /\left\langle k_{T h}^{2}\right\rangle_{f_{h}}}}{\pi\left\langle k_{T h}^{2}\right\rangle_{f_{h}}}, \quad f_{h}^{a}=f_{1, p}^{a}, f_{1, \pi}^{a}, h_{1, p}^{a}, \\
& f_{h}^{a}\left(x_{h}, \boldsymbol{k}_{T h}, Q_{0}, Q_{0}^{2}\right)=f_{h}^{(1) a}\left(x_{h}, Q_{0}\right) \frac{2 M_{h}^{2}}{\pi\left\langle k_{T h}^{2}\right\rangle_{f_{h}}^{2}} e^{-\boldsymbol{k}_{T h}^{2} /\left\langle k_{T h}^{2}\right\rangle_{f_{h}}}, \quad f_{h}^{a}=f_{1 T, p}^{\perp a}, h_{1, p}^{\perp a}, h_{1, \pi}^{\perp a}, h_{1 L, p}^{\perp a}, \\
& f_{h}^{a}\left(x_{h}, \boldsymbol{k}_{T h}, Q_{0}, Q_{0}^{2}\right)=f_{h}^{(2) a}\left(x_{h}, Q_{0}\right) \frac{2 M_{h}^{4}}{\pi\left\langle k_{T h}^{2}\right\rangle_{f_{h}}^{3}} e^{-\boldsymbol{k}_{T h}^{2} /\left\langle k_{T h}^{2}\right\rangle_{f_{h}}}, \quad f_{h}^{a}=h_{1 T, p}^{\perp a},
\end{aligned}
$$

where transverse moments of TMDs are defined in eq. (2.17). Parametrizations from eqs. (2.27) are often used in phenomenology to describe polarized SIDIS and DY data. 
These parametrizations correspond to the following $b_{T}$-space expressions

$$
\begin{array}{rlrl}
\tilde{f}_{h}^{a}\left(x_{h}, b_{T}, Q_{0}, Q_{0}^{2}\right) & =f_{h}^{a}\left(x_{h}, Q_{0}\right) e^{-\frac{1}{4} b_{T}^{2}\left\langle k_{T h}^{2}\right\rangle_{h}}, & \tilde{f}_{h}^{a} & =\tilde{f}_{1, p}^{a}, \tilde{f}_{1, \pi}^{a}, \tilde{h}_{1, p}^{a}, \\
\tilde{f}_{h}^{(1) a}\left(x_{h}, b_{T}, Q_{0}, Q_{0}^{2}\right) & =f_{h}^{(1) a}\left(x_{h}, Q_{0}\right) e^{-\frac{1}{4} b_{T}^{2}\left\langle k_{T h}^{2}\right\rangle_{f_{h}},}, \tilde{f}_{h}^{(1) a}=\tilde{f}_{1 T, p}^{\perp(1) a}, \tilde{h}_{1, p}^{\perp(1) a}, \tilde{h}_{1, \pi}^{\perp(1) a}, \tilde{h}_{1 L, p}^{\perp(1) a}, \\
\tilde{f}_{h}^{(2) a}\left(x_{h}, b_{T}, Q_{0}, Q_{0}^{2}\right) & =f_{h}^{(2) a}\left(x_{h}, Q_{0}\right) e^{-\frac{1}{4} b_{T}^{2}\left\langle k_{T h}^{2}\right\rangle_{f_{h}},}, \tilde{f}_{h}^{(2) a}=\tilde{h}_{1 T, p}^{\perp(2) a},
\end{array}
$$

where the collinear functions are the same as in eqs. (2.27).

Using eqs. (2.27) or eqs. (2.28) one obtains for the convolution integrals in eqs. (2.1) or eqs. (2.9)-(2.14) the following results at the initial scale $Q_{0}$,

$$
\begin{aligned}
F_{U U}^{1}\left(x_{\pi}, x_{p}, q_{T}, Q_{0}^{2}\right)= & \frac{1}{N_{c}} \sum_{a} e_{a}^{2} f_{1, \pi}^{\bar{a}}\left(x_{\pi}, Q_{0}\right) f_{1, p}^{a}\left(x_{p}, Q_{0}\right) \frac{e^{-q_{T}^{2} /\left\langle q_{T}^{2}\right\rangle}}{\pi\left\langle q_{T}^{2}\right\rangle}, \\
F_{U T}^{\sin \phi_{S}}\left(x_{\pi}, x_{p}, q_{T}, Q_{0}^{2}\right)= & \frac{1}{N_{c}} \sum_{a} e_{a}^{2} f_{1, \pi}^{\bar{a}}\left(x_{\pi}, Q_{0}\right) f_{1 T, p}^{\perp(1) a}\left(x_{p}, Q_{0}\right) 2 M_{p} \frac{q_{T}}{\left\langle q_{T}^{2}\right\rangle} \frac{e^{-q_{T}^{2} /\left\langle q_{T}^{2}\right\rangle}}{\pi\left\langle q_{T}^{2}\right\rangle}, \\
F_{U T}^{\sin \left(2 \phi-\phi_{S}\right)}\left(x_{\pi}, x_{p}, q_{T}, Q_{0}^{2}\right)= & -\frac{1}{N_{c}} \sum_{a} e_{a}^{2} h_{1, \pi}^{\perp(1) \bar{a}}\left(x_{\pi}, Q_{0}\right) h_{1, p}^{a}\left(x_{p}, Q_{0}\right) 2 M_{\pi} \frac{q_{T}}{\left\langle q_{T}^{2}\right\rangle} \frac{e^{-q_{T}^{2} /\left\langle q_{T}^{2}\right\rangle}}{\pi\left\langle q_{T}^{2}\right\rangle}, \\
F_{U U}^{\cos 2 \phi}\left(x_{\pi}, x_{p}, q_{T}, Q_{0}^{2}\right)= & \frac{1}{N_{c}} \sum_{a} e_{a}^{2} h_{1, \pi}^{\perp(1) \bar{a}}\left(x_{\pi}, Q_{0}\right) h_{1, p}^{\perp(1) a}\left(x_{p}, Q_{0}\right) \\
& \times 4 M_{\pi} M_{p} \frac{q_{T}^{2}}{\left\langle q_{T}^{2}\right\rangle^{2}} \frac{e^{-q_{T}^{2} /\left\langle q_{T}^{2}\right\rangle}}{\pi\left\langle q_{T}^{2}\right\rangle}, \\
F_{U L}^{\sin 2 \phi}\left(x_{\pi}, x_{p}, q_{T}, Q_{0}^{2}\right)= & -\frac{1}{N_{c}} \sum_{a} e_{a}^{2} h_{1, \pi}^{\perp(1) \bar{a}}\left(x_{\pi}, Q_{0}\right) h_{1 L, p}^{\perp(1) a}\left(x_{p}, Q_{0}\right) \\
& \times 4 M_{\pi} M_{p} \frac{q_{T}^{2}}{\left\langle q_{T}^{2}\right\rangle^{2}} \frac{e^{-q_{T}^{2} /\left\langle q_{T}^{2}\right\rangle}}{\pi\left\langle q_{T}^{2}\right\rangle}, \\
F_{U T}^{\sin \left(2 \phi+\phi_{S}\right)}\left(x_{\pi}, x_{p}, q_{T}, Q_{0}^{2}\right)= & -\frac{1}{N_{c}} \sum_{a} e_{a}^{2} h_{1, \pi}^{\perp(1) \bar{a}}\left(x_{\pi}, Q_{0}\right) h_{1 T, p}^{\perp(2) a}\left(x_{p}, Q_{0}\right) \\
& \times 2 M_{\pi} M_{p}^{2} \frac{q_{T}^{3}}{\left\langle q_{T}^{2}\right\rangle^{3}} \frac{e^{-q_{T}^{2} /\left\langle q_{T}^{2}\right\rangle}}{\pi\left\langle q_{T}^{2}\right\rangle},
\end{aligned}
$$

where the index $a=u, \bar{u}, d, \bar{d}, \ldots$ and the mean square transverse momenta $\left\langle q_{T}^{2}\right\rangle$ are defined in each case as the sum of the mean square transverse momenta of the corresponding TMDs; that is in (2.29) in the first equation $\left\langle q_{T}^{2}\right\rangle=\left\langle k_{T \pi}^{2}\right\rangle_{f_{1, \pi}}+\left\langle k_{T p}^{2}\right\rangle_{f_{1, p}}$, in the second equation $\left\langle q_{T}^{2}\right\rangle=\left\langle k_{T \pi}^{2}\right\rangle_{f_{1, \pi}}+\left\langle k_{T p}^{2}\right\rangle_{f_{1 T, p}^{\perp}}$, etc.

In our study we will use the corresponding transverse moments and Gaussian widths from TMD extractions that we will take at the initial scale which corresponds to the $\left\langle Q^{2}\right\rangle$ in the HERMES experiment. We will therefore use $Q_{0}^{2}=2.4 \mathrm{GeV}^{2}$ as our initial scale of TMD evolution in eqs. (2.28) for parametrization of TMDs and in eqs. (2.9)-(2.14) for structure functions that we will evolve to the scale of the COMPASS Drell-Yan measurement.

The scale $Q_{0}^{2}=2.4 \mathrm{GeV}^{2}$ is convenient because parametrizations of many TMDs are available at this scale, and there is a great deal of expertise how to implement CSS evolution starting from $Q_{0}$. However, results from quark models refer to a lower hadronic scale 


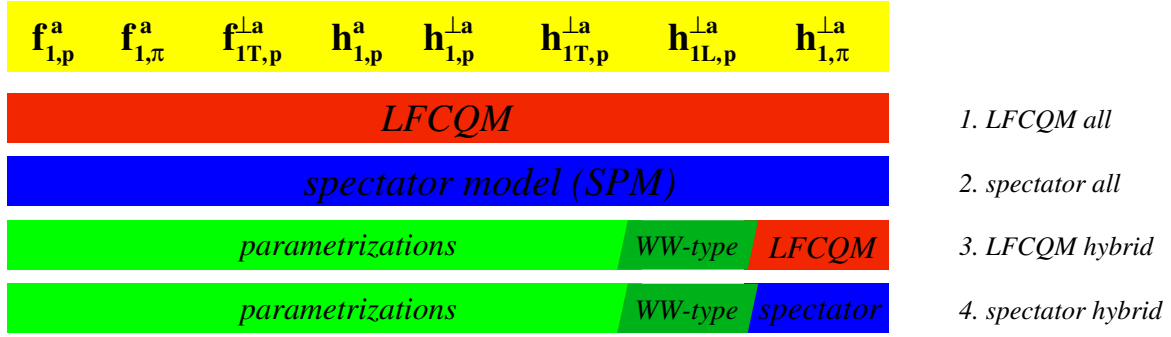

Figure 2. TMDs entering the pion-induced polarized DY process at leading twist in the order from the phenomenologically best to least known, and the approaches used in this work, see text.

$\mu_{0} \sim 0.5 \mathrm{GeV}<Q_{0}$. Presently it is not known how to implement CSS evolution at such low scales (see section 1). Thus, the evolution of model results from $\mu_{0}$ to the initial CSS scale $Q_{0}$ chosen in this work, is regarded as a part of the modelling. It will be described in detail in section 2.5 .

\subsection{TMDs extracted from experimental data}

In order to compute leading-twist structure functions in pion-induced DY the knowledge of the proton and pion TMDs $f_{1, p}^{a}, f_{1, \pi}^{a}, f_{1 T, p}^{\perp a}, h_{1, p}^{a}, h_{1, p}^{\perp a}, h_{1 T, p}^{\perp a}, h_{1 L, p}^{\perp a}, h_{1, \pi}^{\perp a}$ is required, which we list here in the order from the best to the least known TMD, see figure 2 for an overview. While such a classification is to some extent subjective, it is evident that the collinear proton distributions $f_{1, p}^{a}\left(x_{p}\right)$ are the best known [25-28] thanks to DIS, DY and other data. We will utilize the MSTW extraction of $f_{1, p}^{a}\left(x_{p}\right)$ [26] for comparison with models and our calculations. The unpolarized TMDs $f_{1, p}^{a}\left(x_{p}, \boldsymbol{k}_{T p}\right)$ have been studied and much progress was achieved in incorporating effects of QCD evolution [29-33] which are taken into consideration approximately in our approach as described in section 2.2. For the collinear pion distribution $f_{1, \pi}^{a}$, listed next in figure 2, many extractions are available [40-45]. We will use the MRSS fits [41].

One of the most prominent TMDs, the Sivers distribution $f_{1 T, p}^{\perp a}$ was extracted from HERMES, COMPASS, and JLab SIDIS data by several groups with consistent results [34, 38, 127-135]. We will use the extractions of ref. [34] labelled as "Torino" and ref. [38] labelled as "JAM20".

The transversity distribution, $h_{1, p}^{a}$, plays a crucial role in understanding the nucleon spin structure. It is predicted to generate a transverse single spin asymmetry in SIDIS coupling to the Collins fragmentation function [136], which is also responsible for an azimuthal asymmetry in $e^{+} e^{-}$annihilation into hadron pairs. We will use the "Torino" parametrizations of $h_{1, p}^{a}$ from a global QCD analysis of SIDIS and $e^{+} e^{-}$data [35] to be compared with model predictions, and the "JAM20" fit from a global QCD analysis of SIDIS, DY, $e^{+} e^{-}$, and proton-proton data [38] for comparisons and calculations. 
The proton Boer-Mulders function $h_{1, p}^{\perp a}$ extracted from HERMES, COMPASS and DY data in ref. [36] will be used with the label "BMP10." The extraction of $h_{1, p}^{\perp a}$ [36] is less certain, because in SIDIS it requires model-dependent corrections for sizable twist-4 contamination (Cahn effect).

The so-called pretzelosity function $h_{1 T, p}^{\perp a}$ was extracted in ref. [37]. We will label $h_{1 T, p}^{\perp a}$ from ref. [37] as "LP15". Notice that large errors on extracted $h_{1 T, p}^{\perp a}$ were reported in ref. [37]. This is the least known proton TMD for which an extraction has been attempted.

Only the Kotzinian-Mulders distribution $h_{1 L, p}^{\perp a}$ has not yet been extracted. It was found that the data related to this TMD [137-139] are compatible with the WW-type approximation [39] which we will use to approximate $h_{1 L, p}^{\perp a}$ based on $h_{1, p}^{a}$ from "Torino" [35] and "JAM20" [38] fits.

Finally, the pion Boer-Mulders function $h_{1, \pi}^{\perp a}$ is the least known of the TMDs needed to describe the pion-proton DY process at leading twist. No extractions are currently available for this TMD.

\subsection{TMDs from models}

In this section we briefly review the two CQM frameworks, the LFCQM and the SPM, and compare them in figures 3 and 4 to the available phenomenological extractions used in this work.

Light-front models are based on the decomposition of the hadron states in the Fock space constructed in the framework of light-front quantization. The hadron states are then obtained as a superposition of partonic quantum states, each one multiplied by an $N$-parton light-front wave function which gives the probability amplitude to find the corresponding $N$-parton state in the hadron. In the LFCQM the light-front Fock expansion is truncated to the leading component given by the valence $3 q$ and $q \bar{q}$ contribution in the proton and pion, respectively. The light-front wave functions can be further decomposed in terms of lightfront wave amplitudes that are eigenstates of the total parton orbital angular momentum. The TMDs can then be expressed as overlap of light-front wave amplitudes with different orbital angular momentum [54] which makes very transparent the spin-orbit correlations encoded in the different TMDs [54, 55, 57-59]. To model the $3 q$ light-front wave function of the proton, the phenomenological Ansatz of ref. [140] was used, describing the quarkmomentum dependence through a rational analytical expression with parameters fitted to the anomalous magnetic moment of the proton and neutron [140, 141]. For the pion, the $q \bar{q}$ light-front wave function of ref. [142] was used, with the quark-momentum dependent part given by a Gaussian function with parameters fitted to the pion charge radius and decay constant.

Spectator models are based on a field theoretical description of deep inelastic scattering in a relativistic impulse approximation. In this parton model-like factorization, the cross section for deep inelastic scattering processes can be expressed in terms of a Born cross section and quark correlation functions [143]. In this framework, the quark correlation functions are hadronic matrix elements expanded in Dirac and flavor structure multiplying form factors. The essence of the SPMs is to calculate the matrix elements of the quark 

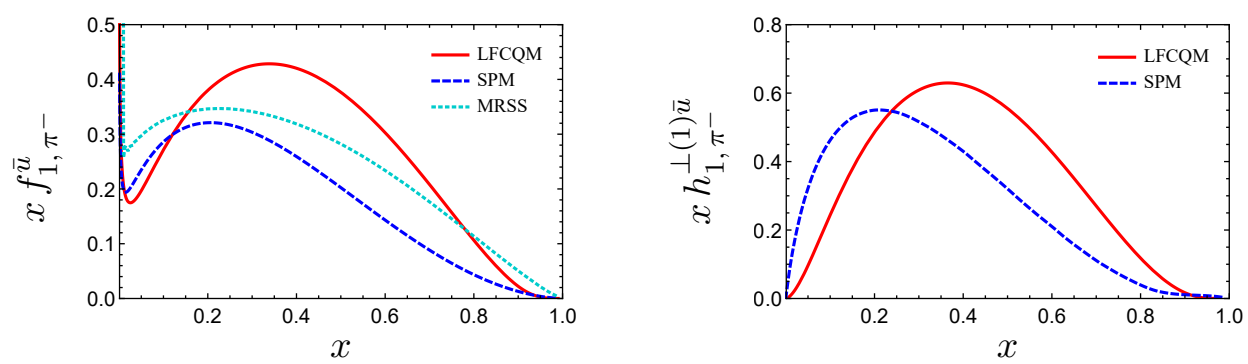

Figure 3. Left: $f_{1, \pi^{-}}^{\bar{u}}$ from LFCQM [59] and SPM [51] LO-evolved to the scale $Q_{0}$ in comparison to MRSS parametrization [41]. Right: predictions from LFCQM [59] and SPM [51] for the pion Boer-Mulders function (with the sign for DY) for which no parametrizations are currently available.

correlation function by the introduction of effective hadron-spectator-quark (e.g. nucleondiquark-quark) vertices $[49,50,144]$ which in turn enable one to model essential nonperturbative flavor and spin structure of hadrons.

The SPMs allow one to model the dynamics of universality and process dependence through studying the gauge-link, and phase content of TMDs [145-151]. In turn systematic phenomenological estimates for parton distributions and fragmentation functions for both "T-even" and "T-odd" TMDs have been carried out [50, 52, 146, 147, 152, 153]. In regard to the latter, it is in this framework that the first calculations of the Sivers and BoerMulders functions of the nucleon were carried out [145-147] and shown on general grounds to contribute to semi-inclusive processes at leading power in the hard scale. Later the Boer-Mulders function of the pion was calculated in ref. [51]. The model parameters are determined by comparing the SPM results for $f_{1, p}^{u}(x)$ and $f_{1, p}^{d}(x)$ to the LO low-scale $\left(\mu_{0}^{2}=0.26 \mathrm{GeV}^{2}\right)$ GRV98 parametrization [25].

The proton TMDs for $u$ - and $d$ - quarks are given by linear combinations of contributions from axial-vector and scalar diquarks assuming $\mathrm{SU}(2)$ flavor symmetry [49, 50].

We choose the scale $Q_{0}^{2}=2.4 \mathrm{GeV}^{2}$ as the initial scale for the CSS evolution. The evolution effects between the initial model scale $\mu_{0} \sim 0.5 \mathrm{GeV}$ and $Q_{0}$ cannot be determined exactly in the CSS formalism, see section 2.3, and they also cannot be neglected. We therefore estimate them as follows. We start with the model predictions for the parton distributions or transverse moments of TMDs as they appear in eq. (2.27) at the initial quark model scale $\mu_{0}$. We evolve them using LO DGLAP evolution to the scale $Q_{0}$. In contrast to CSS, experience with implementing DGLAP evolution at low scales is available $[23-25,40,42]$. Hereby we use exact DGLAP evolution for $f_{1, h}^{a}(x)$ and $h_{1, p}^{a}(x)$. In all other cases we use approximate DGLAP evolution: for the transverse momenta of the proton Sivers function we use the $f_{1, h}^{a}(x)$-nonsinglet evolution shown to lead good results in the LFCQM model study of SIDIS asymmetries [58], while for all the chiral-odd TMDs we assume the DGLAP evolution of transversity $[57,154]$. For the $k_{T}$-dependencies of the TMDs we use the same input from TMD parametrizations as described in eq. (2.27).

The predictions from both models evolved in this way are shown along with the available parametrizations in figures $3-4$ at the scale $Q_{0}$. It is important to stress that in this way we are able to test the $x$-dependencies of the model predictions against the COMPASS 

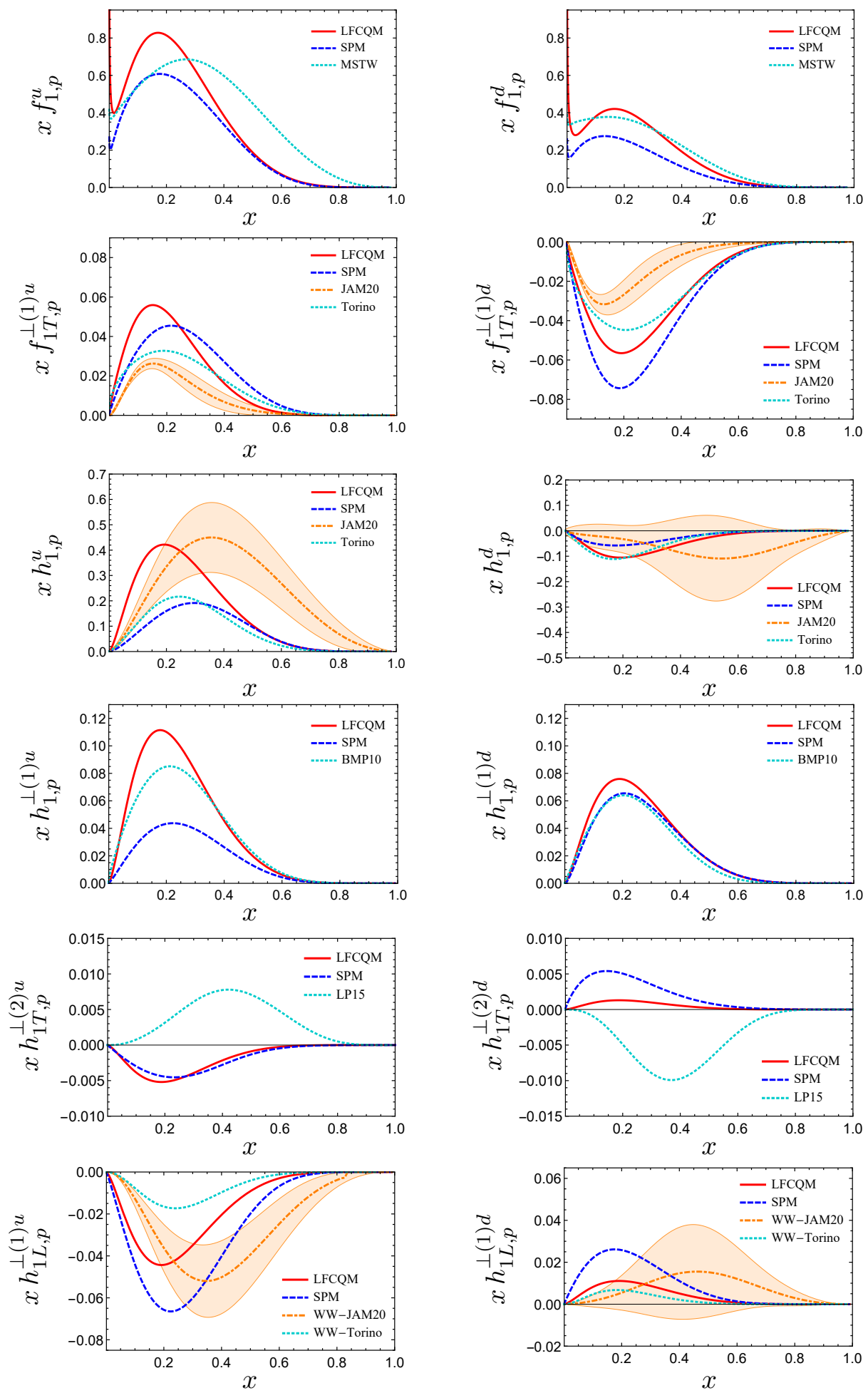

Figure 4. The proton TMDs of $u$ and $d$ quarks in LFCQM $[54,57,58]$ and SPM [50] at the scale $Q_{0}$ compared to phenomenological fits for $f_{1, p}$ from MSTW2008(LO) [26], $f_{1 T, p}^{\perp(1) a}$ from JAM20 [38] and Torino [34], $h_{1, p}^{a}$ from JAM20 [38] and Torino [35], $h_{1, p}^{\perp(1) a}$ from BMP10 [36], $h_{1 T, p}^{\perp(2) a}$ from LP15 [37]. Sivers and Boer-Mulders TMDs are shown with the sign for DY process. The error bands show the 1- $\sigma$ uncertainty of the JAM20 extractions [38]. 
data. The ultimate goal would be to test similarly also the quark model predictions for $k_{T}$-dependencies. This requires an implementation of the CSS evolution starting from low initial scales $\mu_{0}<1 \mathrm{GeV}$ which is beyond the scope of this work, and will be addressed in future studies.

The result from the LFCQM [59] and the SPM [51] for $f_{1, \pi^{-}}^{\bar{u}}(x)$ (which coincides with $f_{1, \pi^{-}}^{d}(x)$ due to isospin symmetry) compare well to the MRSS parametrization [26], see figure 3 . In the region $0.2 \lesssim x_{\pi} \lesssim 0.6$, in which the COMPASS Drell-Yan data points lie, the SPM result agrees within 20-40\% with MRSS [26]. The two models agree well with each other in the case of the pion Boer-Mulders TMD $h_{1, \pi^{-}}^{\perp(1) \bar{u}}(x)=h_{1, \pi^{-}}^{\perp(1) d}(x)$ for which no extraction is available (so far). This robustness of the model predictions is important: the pion Boer-Mulders function enters 4 (out of 6) twist-2 pion-nucleon DY structure functions.

The results from the LFCQM $[54,57,58]$ and the SPM [50] for the proton quark distributions are shown in figure 4 . The region $0.05 \lesssim x_{p} \lesssim 0.4$ is probed in the COMPASS DY measurements [46], see section. 3.1. The model results for the functions $f_{1, p}^{u}(x)$, $f_{1, p}^{d}(x), f_{1 T, p}^{(1) u}(x), f_{1 T, p}^{(1) d}(x), h_{1, p}^{d}(x), h_{1, p}^{\perp(1) d}(x), h_{1 L, p}^{(1) u}(x), h_{1 T, p}^{(2) u}(x)$ agree within $20-40 \%$, and for $h_{1, p}^{u}(x), h_{1, p}^{\perp(1) u}(x), h_{1 L, p}^{(1) d}(x)$ within 40-60\%. Merely for $h_{1 T, p}^{(2) d}(x)$ we observe a more sizable spread of model predictions. In all cases the models agree on the signs of the TMDs. The model results for the unpolarized distributions agree reasonably well with MSTW [26]. The model predictions for transversity and Sivers function are compatible with the corresponding Torino [34, 35] and JAM20 fits [38]. The 1- $\sigma$ uncertainty bands are shown for JAM20 [38]. The corresponding uncertainty bands of the Torino parametrizations [34, 35] are somewhat larger (as more data were used in the JAM20 analysis, cf. section 2.3) and not displayed for better visibility. The proton Boer-Mulders function from models is in good agreement with the BMP10 extraction [36] which has significant statistical and systematic uncertainties, as discussed in section 2.3, and are not shown in figure 4 . The model predictions for pretzelosity show little agreement with the best fit result from LP15 [37], but are within its $1-\sigma$ region which is not shown in the plot.

The comparison in figure 4 indicates an accuracy of the CQMs which is in many cases of the order of 20-40\%. Considering the much different physical foundations of the two models, one may speak about an overall robust CQM picture for the TMDs needed in our work.

\section{Results and observations}

In this section we briefly describe the COMPASS experiment, outline how we explore the model predictions and phenomenological TMD fits, present our results, and compare them to the data.

\subsection{The COMPASS Drell-Yan experiment}

The COMPASS 2015 data [46] were taken with a pion beam of $190 \mathrm{GeV}$ impinging on a transversely polarized $\mathrm{NH}_{3}$ target with a polarization of $\left\langle S_{T}\right\rangle \approx 73 \%$ and a dilution factor $\langle f\rangle \approx 0.18$. The dimuon mass range $4.3 \mathrm{GeV}<Q<8.5 \mathrm{GeV}$ above charmonium resonance 
region but below $\Upsilon$ threshold was covered with the mean value $\langle Q\rangle=5.3 \mathrm{GeV}$. Due to the fixed target kinematics the pion structure was probed at higher $\left\langle x_{\pi}\right\rangle=0.50$ compared to the proton $\left\langle x_{p}\right\rangle=0.17$. The cut $q_{T}>0.4 \mathrm{GeV}$ was imposed and $\left\langle q_{T}\right\rangle=1.2 \mathrm{GeV}$ [46]. The analysis of the data collected by the experiment in 2018 in similar conditions is currently under way [155].

\subsection{The approaches for numerical estimates}

The Sivers asymmetry $A_{U T}^{\sin \phi}$ can be described completely in terms of both, model predictions and available parametrizations, and is the only asymmetry where the latter is possible. For the phenomenological calculation we will use the Torino [35] and JAM20 [38] analysis results for $f_{1 T . p}^{\perp(1) a}(x)$, and MSTW [26] and MRSS [41] parametrizations for proton and pion collinear unpolarized distributions.

The other asymmetries require the knowledge of the pion Boer-Mulders function for which no parametrization is available. In these cases we shall adopt two different main approaches, pure and hybrid, see figure 2 for an overview. We will present therefore up to four different calculations for each observable by exploring the model results and available parametrizations discussed in sections $2.2,2.3,2.4$ and displayed in figures $3-4$. The first approach makes a pure use of model predictions for all pion and proton TMDs which will be labelled in the plots by the acronyms LFCQM or SPM.

In the hybrid-approaches we will use the minimal model input, the predictions from the LFCQM [59] and SPM [51] for the pion Boer-Mulders function, and the maximal input from parametrizations: JAM20 [38] for $f_{1 T, p}^{\perp a}$ and $h_{1, p}^{a}$, BMP10 [36] for $h_{1, p}^{\perp a}$, and LP15 [37] for $h_{1 T, p}^{\perp a}$. The results will be labelled respectively as "LFC-JAM20", "LFCLP15", "LFC-BMP10" or "SPM-JAM20," "SPM-LP15," "SPM-BMP10." For $h_{1 L, p}^{\perp a}$ we make use of WW-type approximation which allows one to approximate this TMD in terms of $h_{1, p}^{a}$ for which we will use JAM20 [38]. WW-type approximations were explored in ref. [39] and shown to work well with the available data. We will add "WW" in the label of calculation when WW approximation is used. For all hybrid calculations we will use the parametrizations $[26,41]$ for $f_{1, p}^{a}$ and $f_{1, \pi}^{a}$.

\subsection{Discussion of the results and comparison to available data}

Numerical results for the leading-twist pion-nucleon DY asymmetries are shown in figures 5-9 in comparison to available COMPASS data. Table 1 gives a detailed overview on the model results and phenomenological information.

Let us start the discussion with the Sivers asymmetry. One of the most striking features of "naively" T-odd (Sivers, Boer-Mulders) TMDs is the expected sign change [156] from SIDIS to DY due to the difference of initial (DY) versus final (SIDIS) state interactions $[153,157]$. Verification of the sign change of the Sivers function is one of the milestones of DY programs of COMPASS and RHIC [158]. In SIDIS the proton $u$-quark Sivers function is negative, while STAR RHIC [159] $W^{ \pm} / Z$ asymmetry data favor a positive sign [160] hinting on the predicted process dependence of T-odd TMDs [156].

The predictions for the $A_{U T}^{\sin \phi_{S}}$ asymmetry at COMPASS are positive, see for instance refs. [161-163]. Our calculations confirm this expectation, see figure 5 where we compare 

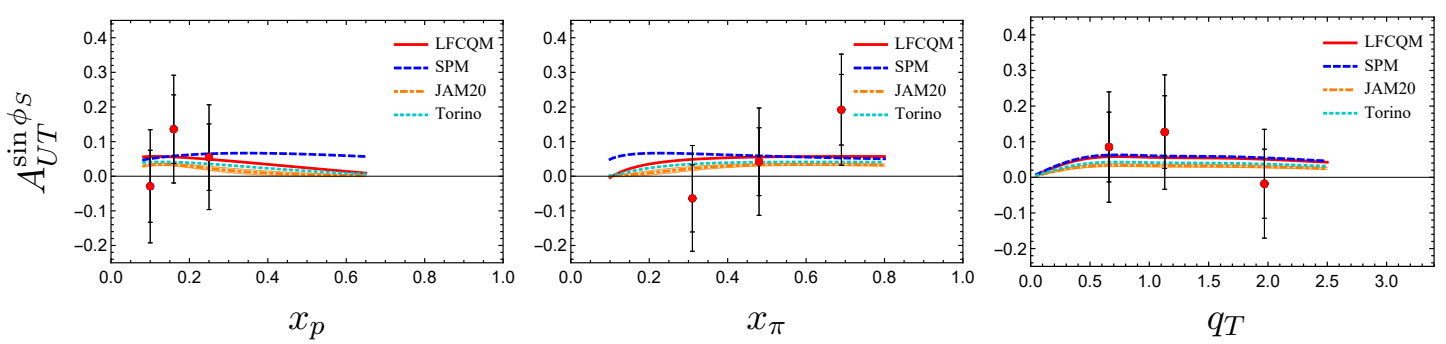

Figure 5. $A_{U T}^{\sin \phi}$ as a function of $x_{p}$ (left), $x_{\pi}$ (middle) and $q_{T}$ (right) vs COMPASS data [46].
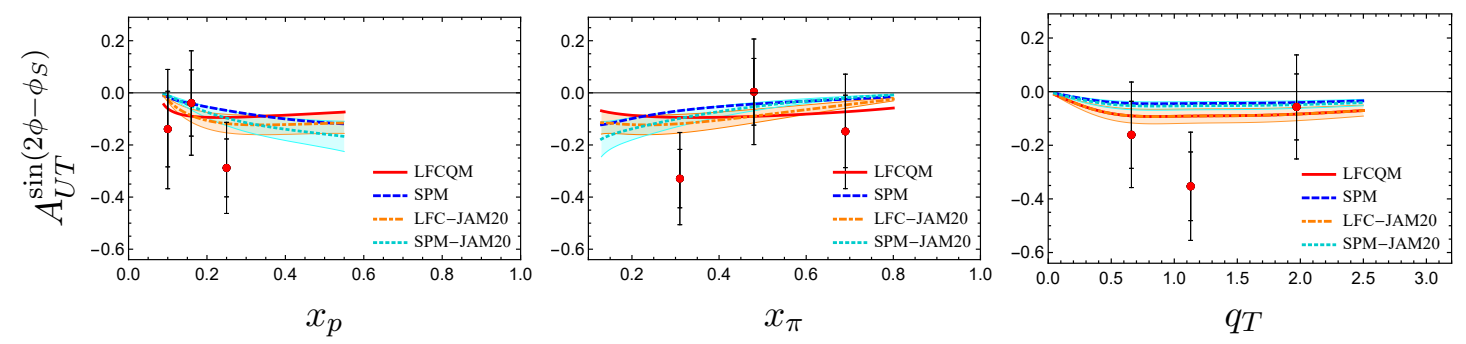

Figure 6. $A_{U T}^{\sin \left(2 \phi-\phi_{S}\right)}$ as a function of $x_{p}$ (left), $x_{\pi}$ (middle) and $q_{T}$ (right) vs COMPASS data [46].
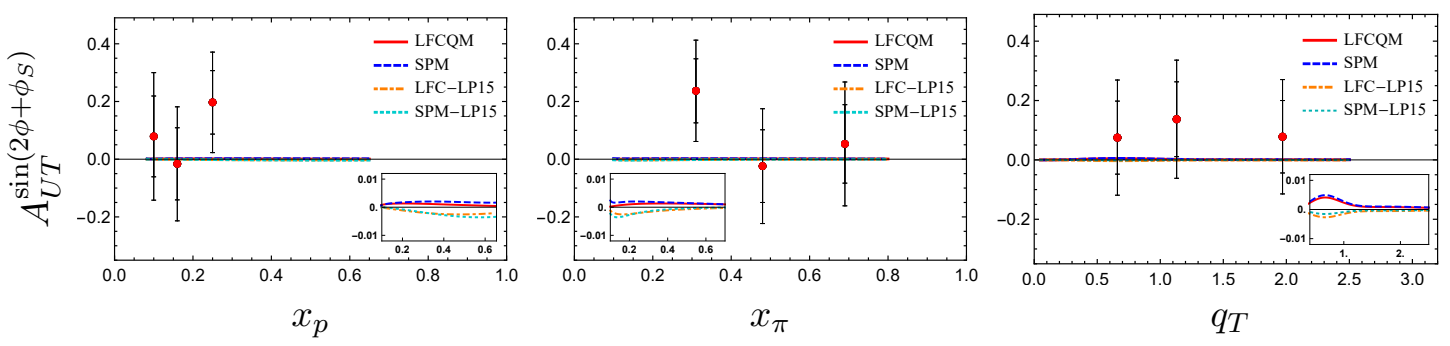

Figure 7. $A_{U T}^{\sin \left(2 \phi+\phi_{S}\right)}$ as a function of $x_{p}$ (left), $x_{\pi}$ (middle) and $q_{T}$ (right) vs COMPASS data [46].

our results to COMPASS data [46]. The $u$-quark Sivers function in DY is expected to be positive, see figure 4. If we disregard sea quark effects, which were shown to play a negligible role in $\pi^{-}$-proton DY in the COMPASS kinematics [162], then $A_{U T}^{\sin \phi_{S}} \propto f_{1 T, p}^{\perp u}\left(x_{p}\right)>0$. The experimental error bars are currently sizeable, but the data show a tendency to positive asymmetry, see figure 5, in agreement with the expected sign change of the Sivers function. Clearly, more experimental evidence is needed to corroborate this finding.

In the global QCD analysis of single-spin asymmetries [38] the COMPASS data [46] were used, such that the JAM20 result in figure 5 is consistent with all present-day data on observables related to Sivers functions. It is worth remarking that predictions based on the earlier Torino extraction [34] (which used SIDIS data only) yield a somewhat larger asymmetry than JAM20 and are closer to the LFCQM and SPM results in figure 5. This result is consistent with the different size of Sivers functions found in ref. [34] and ref. [38], see figure 4 .

Figure 6 shows the asymmetry $A_{U T}^{\sin \left(2 \phi-\phi_{S}\right)}$ which arises from a convolution of transversity and pion Boer-Mulders function in comparison to COMPASS data [46]. In the case 

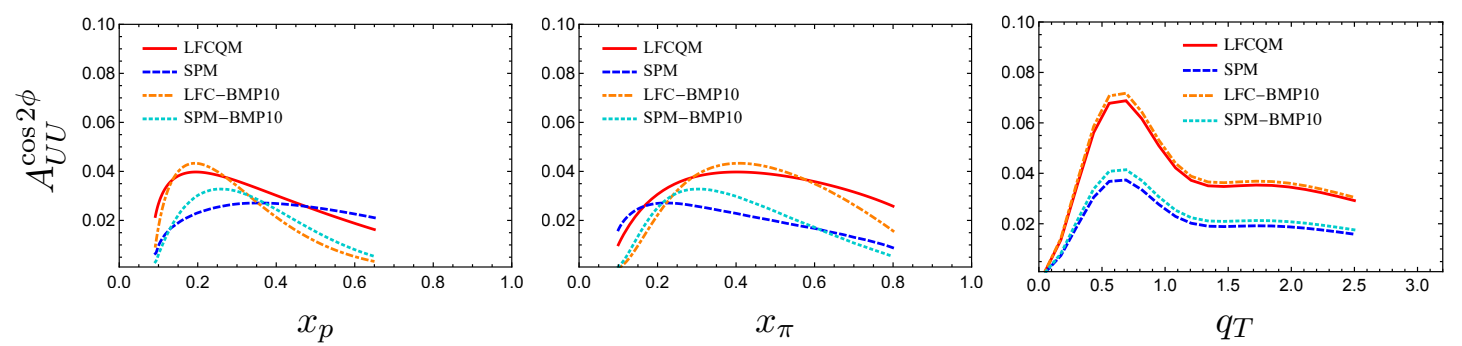

Figure 8. $A_{U U}^{\cos 2 \phi}$ as a function of $x_{p}$ (left), $x_{\pi}$ (middle) and $q_{T}$ (right) in the COMPASS kinematics.
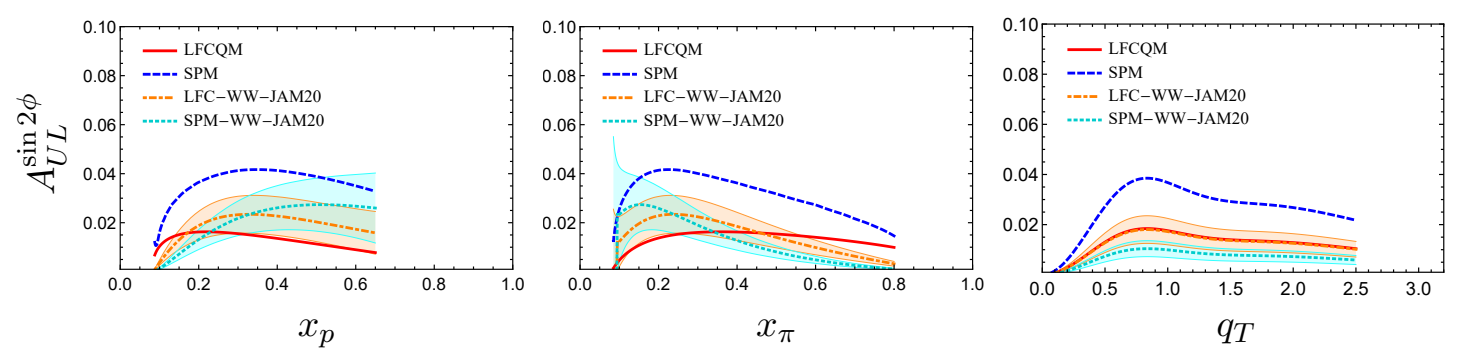

Figure 9. $\quad A_{U L}^{\sin 2 \phi}$ as a function of $x_{p}$ (left), $x_{\pi}$ (middle) and $q_{T}$ (right) in the COMPASS kinematics.

\begin{tabular}{|c|c|c|c|c|c|}
\hline Figure & structure function & TMDs & LFCQM & SPM & phenomenology \\
\hline \hline $5-9$ & $F_{U U}^{1}$ & $f_{1, p}^{a}, \quad f_{1, \pi}^{a}$ & {$[54,59]$} & {$[50,51]$} & {$[26,41]$} \\
\hline 5 & $F_{U T}^{\sin \phi_{S}}$ & $f_{1 T, p}^{\perp a}, f_{1, \pi}^{a}$ & {$[55,59]$} & {$[50,51]$} & {$[38,41]$} \\
\hline 6 & $F_{U T}^{\sin \left(2 \phi-\phi_{S}\right)}$ & $h_{1, p}^{a}, h_{1, \pi}^{\perp a}$ & {$[54,59]$} & {$[50,51]$} & {$[38],-$} \\
\hline 7 & $F_{U T}^{\sin \left(2 \phi+\phi_{S}\right)}$ & $h_{1 T, p}^{\perp a}, h_{1, \pi}^{\perp a}$ & {$[54,59]$} & {$[49,51]$} & {$[37],-$} \\
\hline 8 & $F_{U U}^{\cos 2 \phi}$ & $h_{1, p}^{\perp a}, h_{1, \pi}^{\perp a}$ & {$[55,59]$} & {$[50,51]$} & {$[36],-$} \\
\hline 9 & $F_{U L}^{\sin 2 \phi}$ & $h_{1 L, p}^{\perp a}, h_{1, \pi}^{\perp a}$ & {$[54,59]$} & {$[50,51]$} & {$[39],-$} \\
\hline
\end{tabular}

Table 1. Overview on non-perturbative input used to produce the results in figures 5-9 which was taken from the LFCQM, the SPM, and phenomenological fits (or WW-type approximation in the case of $h_{1 L, p}^{\perp a}$ ). Notice that no phenomenological information is currently available on $h_{1, \pi}^{\perp a}$, cf. section 2.3 .

of this asymmetry the pure model and hybrid calculations yield results in good mutual agreement. Neglecting sea quarks, it is $A_{U T}^{\sin \left(2 \phi-\phi_{S}\right)} \propto-h_{1, \pi^{-}}^{\perp(1) \bar{u}}\left(x_{\pi}\right) h_{1, p}^{u}\left(x_{p}\right)<0$. Both, $h_{1, \pi^{-}}^{\perp(1) \bar{u}}$ and $h_{1, p}^{u}$ are positive, see figure 4 , and we predict a negative asymmetry. This is consistent with the trend of the data. We therefore conclude that the COMPASS data [46] indicate a positive sign for the pion Boer-Mulders TMD $h_{1, \pi^{-}}^{\perp(1)}$. (It is important to recall that absolute signs in extractions of chiral-odd TMDs and fragmentation functions are convention-dependent because chiral-odd functions contribute to observables always in connection with other chiral-odd functions. The convention used for TMD extractions 
is $h_{1, p}^{u}(x)>0$. This sign is a choice which is well-informed by model and lattice QCD calculations but not an experimental observation.) The indication that $h_{1, \pi^{-}}^{\perp(1) \bar{u}}>0$ is an important result which can be used to test the process dependence of the proton BoerMulders function, see below.

Figure 7 shows $A_{U T}^{\sin \left(2 \phi+\phi_{S}\right)}$ which is due to the convolution of pretzelosity and pion Boer-Mulders function compared to COMPASS data [46]. This asymmetry is proportional to $q_{T}^{3}$ for $q_{T} \ll 1 \mathrm{GeV}$. This leads to a kinematic suppression of this asymmetry as compared to the two previous asymmetries (both proportional to $q_{T}$ at small transverse momenta). As a consequence $A_{U T}^{\sin \left(2 \phi+\phi_{S}\right)}$ is by far the smallest of the leading-twist asymmetries in pion-nucleon DY. Numerically it is $1 \%$ or smaller, such that we had to include the insets in figure 7 to display the theoretical curves. The LFCQM and the SPM are in good agreement with each other, but not with the LP15 fit of pretzelosity [37] which suggests an opposite sign for the asymmetry. At this point one has to stress that the LP15 fit of [37] has a large statistical uncertainty (not displayed in figures 4 and 7 ) and is compatible with zero or opposite sign within 1- $\sigma$. This TMD is difficult to measure in DY and SIDIS. In the high luminosity SIDIS experiments at JLab $12 \mathrm{GeV}$ and the future Electron Ion Collider it may be feasible to measure pretzelosity.

The $A_{U U}^{\cos 2 \phi}$ asymmetry in unpolarized DY originates from a convolution of the BoerMulders functions in nucleon and pion. Historically it was connected to the "violation" of the Lam-Tung relation, see [164] and references therein. A simultaneous measurement of $A_{U U}^{\cos 2 \phi}$ and $A_{U T}^{\sin \left(2 \phi-\phi_{S}\right)}$ which we have discussed above allows one to test the sign change of the proton Boer-Mulders function in DY. $A_{U U}^{\cos 2 \phi}$ was measured and found positive in earlier CERN and Fermilab measurements $[165,166]$. Neglecting sea quark effects, the asymmetry is dominated by $A_{U U}^{\cos 2 \phi} \propto h_{1, \pi^{-}}^{\perp(1) \bar{u}}\left(x_{\pi}\right) h_{1, p}^{\perp(1) u}\left(x_{p}\right)$. With the indication of the positive sign for the pion Boer-Mulders function from the COMPASS data [46] on $A_{U T}^{\sin \left(2 \phi-\phi_{S}\right)}$, we conclude a positive sign also for the proton $u$-quark Boer-Mulders function in DY, which is opposite to the sign seen in SIDIS analyses [167] and hence in agreement with the prediction for the process dependence property of T-odd TMDs [156].

Figure 8 shows our predictions for $A_{U U}^{\cos 2 \phi}$ for COMPASS kinematics. At this point no data are available from COMPASS, but an analysis is planned [168] and our predictions in figure 8 may be tested in near future. It is worth recalling that our approach provides a good description of the NA10 CERN [165] and E615 Fermilab [166] data. The test of our predictions in figure 8 will help to investigate the compatibility of the NA10, E615 and COMPASS experiments. Interestingly, fixed-order collinear factorized perturbative QCD calculations, which strictly speaking require $q_{T}$ to be the hard scale, can also qualitatively describe the NA10 and E615 data [69, 70]. It will be interesting to confront those calculations with future COMPASS data and TMD studies.

Notice that in the analysis [167] of the proton-proton and proton-deuteron data from the FNAL E866/NuSea experiment [169, 170] indications were obtained that the proton quark and antiquark Boer-Mulders functions (in DY) have the same signs. With our observations based on COMPASS data we therefore infer a first hint that also the BoerMulders functions of $\bar{u}$ and $\bar{d}$ are positive in DY. Interestingly, not only valence Boer- 
Mulders distributions in nucleon and pion seem "alike" [171], but also the nucleon sea quark distributions seem to have all the same sign. This confirms an early estimate on the sign of the anti-quark Boer Mulders function carried in the SPM in ref. [143]. This is in line with predictions from the limit of a large number of colors $N_{c}$ in QCD that $h_{1, p}^{\perp u}\left(x_{p}, \boldsymbol{k}_{T p}\right)=$ $h_{1, p}^{\perp d}\left(x_{p}, \boldsymbol{k}_{T p}\right)$ and $h_{1, p}^{\perp \bar{u}}\left(x_{p}, \boldsymbol{k}_{T p}\right)=h_{1, p}^{\perp \bar{d}}\left(x_{p}, \boldsymbol{k}_{T p}\right)$ modulo $1 / N_{c}$ corrections [172]. Future data will provide more stringent tests of these predictions.

Finally, it is worth pointing out that in principle one can extract the $u$-quark transversity distribution entirely from the measurements of $A_{U U}^{\cos 2 \phi}$ and $A_{U T}^{\sin \left(2 \phi-\phi_{S}\right)}$ in $\pi^{-}$-proton DY at COMPASS [173]. While typically data available from different processes are processed in "global analyses," whenever possible it is also valuable to extract a function from one process alone. This would for instance allow one to test the universality (same sign and $x$-shape in SIDIS and DY) of the $u$-quark transversity distribution which is otherwise taken for granted.

Figure 9 displays our predictions for the longitudinal single-spin asymmetry $A_{U L}^{\sin 2 \phi}$ in the COMPASS kinematics which is due to the Kotzinian-Mulders TMD $h_{1 L}^{\perp a}$ and the pion Boer-Mulders function. If we disregard sea quark effects, then $A_{U L}^{\sin 2 \phi} \propto$ $-h_{1, \pi^{-}}^{\perp(1) \bar{u}}\left(x_{\pi}\right) h_{1 L, p}^{\perp(1) u}\left(x_{p}\right)>0$. Especially the SPM predicts a sizable and positive asymmetry. Since no parametrization on $h_{1 L}^{\perp a}$ is currently available, the hybrid calculations make use of the WW-type approximation which is compatible with SIDIS data [39]. This is the only leading-twist pion-proton asymmetry in DY which requires a longitudinal proton polarization. We are not aware of plans to run DY experiments with longitudinal proton polarization in the near future. Potentially $A_{U L}^{\sin 2 \phi}$ could be studied in DY with doubly polarized protons or deuterons in a future NICA experiment [174].

We also study the theoretical uncertainty due to the variation of $C_{1}$ and $C_{2}$ in eqs. (2.5), (2.18), and (2.24) at NLL accuracy. Such studies are of importance in order to establish the control over the perturbative expansion, see e.g. [109]. We will use $A_{U U}^{\cos 2 \phi}$ asymmetry as an example. Scale dependence on $C_{2}$ cancels exactly at this order between the numerator and the denominator of the asymmetry. In figure 10 we show the corresponding theoretical uncertainty due to variation of $C_{1} \in\left[e^{-\gamma_{E}}, 4 e^{-\gamma_{E}}\right]$ for the LFCQM, notice that for $C_{1}=e^{-\gamma_{E}}$ the asymmetry becomes larger than the red curve calculated with $C_{1}=2 e^{-\gamma_{E}}$, while for $C_{1}=4 e^{-\gamma_{E}}$ the value of asymmetry decreases very slightly. One can see that the theoretical uncertainty due to the scale choice of $C_{1}$ is not negligible and warrants the inclusion of higher order corrections in the calculations. This uncertainty is smaller than the spread of the model predictions shown in figure 8 and therefore we expect that the future data will be able to distinguish among various models.

Before ending this section it is important to remark that the COMPASS experiment has covered the range $0.4 \mathrm{GeV}<q_{T}<5 \mathrm{GeV}$. At the upper limit the condition $q_{T} \ll Q$ for the applicability of the TMD factorization is not satisfied which constitutes an uncertainty in our calculations. However, in the experiment (and in our calculations) it is $\left\langle q_{T}\right\rangle=1.2 \mathrm{GeV}$ which is much smaller than $\langle Q\rangle=5.3 \mathrm{GeV}$ and we verified that the region of large $q_{T}$ (namely, $3 \mathrm{GeV}<q_{T}<5 \mathrm{GeV}$ ) in our calculations has a negligible impact on the $q_{T^{-}}$ averaged (integrated) asymmetries in the experiment. 


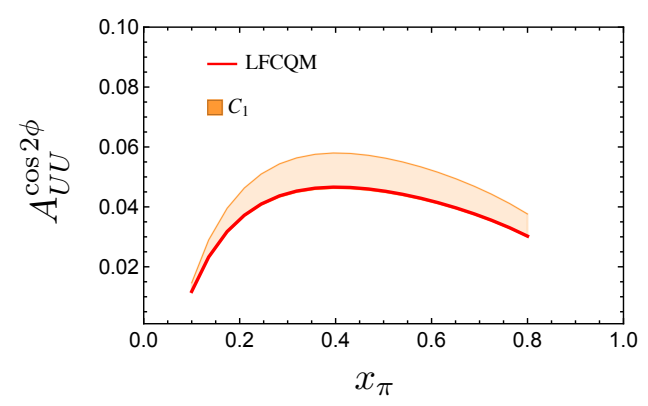

Figure 10. $A_{U U}^{\cos 2 \phi}$ as a function of $x_{\pi}$, the orange region corresponds to variation of $C_{1}$ in the interval $\left[e^{-\gamma_{E}}, 4 e^{-\gamma_{E}}\right]$ and illustrates the sensitivity of our results to scale variations.

\section{Conclusions}

In this work we studied the DY process with negative pions and polarized protons with focus on the kinematics of the COMPASS experiment. As no phenomenological extractions are available for the Boer-Mulders TMD function of the pion, we explored two popular and widely used hadronic models, the LFCQM and the SPM, together with available phenomenological information on the other TMDs. For the LFCQM and the SPM we implement TMD evolution at NLL accuracy from fixed scale according to the solution to the CSS equations in ref. [21] and outlined in section 2.2. This approach moves beyond the approximate TMD evolution based on the Gaussian Ansatz for transverse parton momenta with energy dependent Gaussian widths.

We presented a complete description of polarized DY at leading twist using TMD evolution at NLL accuracy. The required TMDs include on the nucleon side $f_{1, p}^{a}, f_{1 T, p}^{\perp a}$, $h_{1, p}^{a}, h_{1, p}^{\perp a}, h_{1 T, p}^{\perp a}, h_{1 L, p}^{\perp a}$; and on the pion side $f_{1, \pi}^{a}, h_{1, \pi}^{\perp a}$. For that we compiled results from several prior LFCQM and SPM calculations, which to the best of our knowledge have not been presented in this completeness before [49-51, 54, 55, 59]. Based on concise comparisons of model results with available phenomenological information [26, 34-39, 41], we estimate an accuracy of the model results of $20-40 \%$ for the majority of (though not all) TMDs. Similar "model accuracies" were found in prior phenomenological applications of CQMs [57-59].

Driven by the motivation to make maximal use of currently available phenomenological information [26, 34-39, 41], we also carried out "hybrid" calculations with a minimal model dependence - namely only due to the pion Boer-Mulders function for which no extraction is currently available. In this way we provided up to four predictions for each DY observable, with different levels of model dependence. The critical comparison of the various results (pure-model and hybrid calculations in respectively LFCQM and SPM) allows us to differentiate robust predictions from more strongly model-dependent results.

Our study had two main goals, namely to present theoretical calculations which help to interpret the first data from the pion-induced DY with polarized protons measured by COMPASS, as well as to provide quantitative tests of the application of CQMs to the description of pion and nucleon structure. 
In regard to the interpretation of the first data from the pion-induced DY with polarized protons, we observe a robust picture. The pure-model and hybrid calculations from the LFCQM and SPM are in remarkable agreement with each other at the present stage. The theoretical spread of our results is smaller than the present uncertainties of the available data. Among the most interesting observations are the encouraging indications for the change of sign of the T-odd TMDs in DY vs SIDIS, both in the case of the proton Sivers and proton Boer-Mulders function. These are model independent results. Another modelindependent result is the observation that the data favor a positive (in DY) Boer-Mulders $\bar{u}$-distribution in $\pi^{-}$. We also report the first indication that all proton Boer-Mulders functions for $u, d, \bar{u}, \bar{d}$ flavors are positive (in DY). At the present, these observations are admittedly vague due to the low precision of the current data. More precise future data from COMPASS and other facilities will allow us to solidify the picture.

In regard to the quantitative tests of the application of CQMs, it is important to stress that the DY process with $\pi^{-}$and proton in the COMPASS kinematics is an ideal process for these purposes. In the COMPASS kinematics sea quarks do not play an important role [162]. Due to the $u$-quark dominance in the proton the process is strongly dominated by annihilations of $\bar{u}$ from $\pi^{-}$and $u$ from proton in the valence $x$-region where CQMs can be expected to catch the main features in the hadronic structure of the pion and nucleon.

CQMs are important qualitative tools for QCD calculations. Within their model accuracy and within their range of applicability in the valence $x$-region, we observe that CQMs yield useful results and provide helpful guidelines for the interpretation of data. Future data will provide more stringent tests of the CQMs, and allow for extraction of hadron structure by global QCD analyses. We also provided several predictions that await experimental confirmation.

\section{Acknowledgments}

The authors wish to thank A. V. Efremov and A. Kotzinian for valuable discussions which motivated this study and J. Collins, T. Rogers, and Z. Kang for discussions on implementation of TMD evolution. This work was supported by the National Science Foundation under the Contracts No. PHY-1812423 (S.B. and P.S.) and No. PHY-2012002 (A.P.), and in part by the US Department of Energy under contracts, No. DE-FG02-07ER41460 (L.G.) and No. DE-AC05-06OR23177 (A.P.) under which JSA, LLC operates JLab, the framework of the TMD Topical Collaboration (L.G. and A.P.), and by the European Union's Horizon 2020 program under grant agreement No. 824093(STRONG2020) (B.P.).

Open Access. This article is distributed under the terms of the Creative Commons Attribution License (CC-BY 4.0), which permits any use, distribution and reproduction in any medium, provided the original author(s) and source are credited.

\section{References}

[1] J.C. Collins, D.E. Soper and G.F. Sterman, Transverse Momentum Distribution in Drell-Yan Pair and W and Z Boson Production, Nucl. Phys. B 250 (1985) 199 [INSPIRE]. 
[2] J. Collins, Foundations of perturbative QCD, Cambridge Monographs on Particle Physics, Nuclear Physics and Cosmology. Vol. 32, Cambridge University Press, Cambridge U.K. (2013).

[3] D.W. Sivers, Single Spin Production Asymmetries from the Hard Scattering of Point-Like Constituents, Phys. Rev. D 41 (1990) 83 [InSPIRE].

[4] M. Anselmino, M. Boglione and F. Murgia, Single spin asymmetry for $p$ (polarized) $p \rightarrow \pi X$ in perturbative QCD, Phys. Lett. B 362 (1995) 164 [hep-ph/9503290] [INSPIRE].

[5] P.J. Mulders and R.D. Tangerman, The Complete tree level result up to order $1 / Q$ for polarized deep inelastic leptoproduction, Nucl. Phys. B 461 (1996) 197 [Erratum ibid. 484 (1997) 538] [hep-ph/9510301] [INSPIRE].

[6] R.N. Cahn, Azimuthal Dependence in Leptoproduction: A Simple Parton Model Calculation, Phys. Lett. B 78 (1978) 269 [INSPIRE].

[7] A. Kotzinian, New quark distributions and semiinclusive electroproduction on the polarized nucleons, Nucl. Phys. B 441 (1995) 234 [hep-ph/9412283] [INSPIRE].

[8] A.M. Kotzinian and P.J. Mulders, Longitudinal quark polarization in transversely polarized nucleons, Phys. Rev. D 54 (1996) 1229 [hep-ph/9511420] [INSPIRE].

[9] A. Bacchetta, M. Diehl, K. Goeke, A. Metz, P.J. Mulders and M. Schlegel, Semi-inclusive deep inelastic scattering at small transverse momentum, JHEP 02 (2007) 093 [hep-ph/0611265] [INSPIRE].

[10] R.D. Tangerman and P.J. Mulders, Intrinsic transverse momentum and the polarized Drell-Yan process, Phys. Rev. D 51 (1995) 3357 [hep-ph/9403227] [InSPIRE].

[11] D. Boer and P.J. Mulders, Time reversal odd distribution functions in leptoproduction, Phys. Rev. D 57 (1998) 5780 [hep-ph/9711485] [INSPIRE].

[12] S. Arnold, A. Metz and M. Schlegel, Dilepton production from polarized hadron hadron collisions, Phys. Rev. D 79 (2009) 034005 [arXiv: 0809.2262] [InSPIRE].

[13] A. Metz and A. Vossen, Parton Fragmentation Functions, Prog. Part. Nucl. Phys. 91 (2016) 136 [arXiv: 1607.02521] [INSPIRE].

[14] J.C. Collins and D.E. Soper, Back-To-Back Jets in QCD, Nucl. Phys. B 193 (1981) 381 [Erratum ibid. 213 (1983) 545] [INSPIRE].

[15] J.-w. Qiu and G.F. Sterman, Single transverse spin asymmetries, Phys. Rev. Lett. 67 (1991) 2264 [INSPIRE].

[16] X.-d. Ji, J.-p. Ma and F. Yuan, QCD factorization for semi-inclusive deep-inelastic scattering at low transverse momentum, Phys. Rev. D 71 (2005) 034005 [hep-ph/0404183] [INSPIRE].

[17] X. Ji, J.-W. Qiu, W. Vogelsang and F. Yuan, Single-transverse spin asymmetry in semi-inclusive deep inelastic scattering, Phys. Lett. B 638 (2006) 178 [hep-ph/0604128] [INSPIRE].

[18] X. Ji, J.-w. Qiu, W. Vogelsang and F. Yuan, Single Transverse-Spin Asymmetry in Drell-Yan Production at Large and Moderate Transverse Momentum, Phys. Rev. D 73 (2006) 094017 [hep-ph/0604023] [INSPIRE].

[19] S.M. Aybat and T.C. Rogers, TMD Parton Distribution and Fragmentation Functions with QCD Evolution, Phys. Rev. D 83 (2011) 114042 [arXiv:1101.5057] [InSPIRE]. 
[20] J.P. Ma and G.P. Zhang, QCD Corrections of All Structure Functions in Transverse Momentum Dependent Factorization for Drell-Yan Processes, JHEP 02 (2014) 100 [arXiv: 1308.2044] [INSPIRE].

[21] J. Collins and T. Rogers, Understanding the large-distance behavior of transverse-momentum-dependent parton densities and the Collins-Soper evolution kernel, Phys. Rev. D 91 (2015) 074020 [arXiv:1412.3820] [InSPIRE].

[22] J. Collins, L. Gamberg, A. Prokudin, T.C. Rogers, N. Sato and B. Wang, Relating Transverse Momentum Dependent and Collinear Factorization Theorems in a Generalized Formalism, Phys. Rev. D 94 (2016) 034014 [arXiv: 1605.00671] [InSPIRE].

[23] M. Gluck, E. Reya and A. Vogt, Parton distributions for high-energy collisions, Z. Phys. C 53 (1992) 127 [INSPIRE].

[24] M. Gluck, E. Reya and A. Vogt, Dynamical parton distributions of the proton and small $x$ physics, Z. Phys. C 67 (1995) 433 [INSPIRE].

[25] M. Glück, E. Reya and A. Vogt, Dynamical parton distributions revisited, Eur. Phys. J. C 5 (1998) 461 [hep-ph/9806404] [INSPIRE].

[26] A.D. Martin, W.J. Stirling, R.S. Thorne and G. Watt, Parton distributions for the LHC, Eur. Phys. J. C 63 (2009) 189 [arXiv:0901.0002] [InSPIRE].

[27] L.A. Harland-Lang, A.D. Martin, P. Motylinski and R.S. Thorne, Parton distributions in the LHC era: MMHT 2014 PDFs, Eur. Phys. J. C 75 (2015) 204 [arXiv:1412.3989] [INSPIRE].

[28] S. Dulat et al., New parton distribution functions from a global analysis of quantum chromodynamics, Phys. Rev. D 93 (2016) 033006 [arXiv:1506.07443] [INSPIRE].

[29] F. Landry, R. Brock, P.M. Nadolsky and C.P. Yuan, Tevatron Run-1 Z boson data and Collins-Soper-Sterman resummation formalism, Phys. Rev. D 67 (2003) 073016 [hep-ph/0212159] [INSPIRE].

[30] M. Anselmino, M. Boglione, J.O. Gonzalez Hernandez, S. Melis and A. Prokudin, Unpolarised Transverse Momentum Dependent Distribution and Fragmentation Functions from SIDIS Multiplicities, JHEP 04 (2014) 005 [arXiv: 1312.6261] [INSPIRE].

[31] A. Signori, A. Bacchetta, M. Radici and G. Schnell, Investigations into the flavor dependence of partonic transverse momentum, JHEP 11 (2013) 194 [arXiv:1309.3507] [INSPIRE].

[32] A. Bacchetta et al., Transverse-momentum-dependent parton distributions up to $N^{3} L L$ from Drell-Yan data, JHEP 07 (2020) 117 [arXiv: 1912.07550] [INSPIRE].

[33] I. Scimemi and A. Vladimirov, Non-perturbative structure of semi-inclusive deep-inelastic and Drell-Yan scattering at small transverse momentum, JHEP 06 (2020) 137 [arXiv: 1912.06532] [INSPIRE].

[34] M. Anselmino, M. Boglione, U. D'Alesio, S. Melis, F. Murgia and A. Prokudin, Sivers Distribution Functions and the Latest SIDIS Data, in 19th International Workshop on Deep-Inelastic Scattering and Related Subjects, Newport News U.S.A. (2011) [arXiv: 1107.4446] [INSPIRE].

[35] M. Anselmino, M. Boglione, U. D’Alesio, S. Melis, F. Murgia and A. Prokudin, Simultaneous extraction of transversity and Collins functions from new SIDIS and $e^{+} e^{-}$ data, Phys. Rev. D 87 (2013) 094019 [arXiv:1303.3822] [INSPIRE]. 
[36] V. Barone, S. Melis and A. Prokudin, The Boer-Mulders effect in unpolarized SIDIS: An Analysis of the COMPASS and HERMES data on the cos 2 phi asymmetry, Phys. Rev. D 81 (2010) 114026 [arXiv:0912.5194] [INSPIRE].

[37] C. Lefky and A. Prokudin, Extraction of the distribution function $h_{1 T}^{\perp}$ from experimental data, Phys. Rev. D 91 (2015) 034010 [arXiv:1411.0580] [InSPIRE].

[38] Jefferson Lab Angular Momentum collaboration, Origin of single transverse-spin asymmetries in high-energy collisions, Phys. Rev. D 102 (2020) 054002 [arXiv: 2002.08384] [INSPIRE].

[39] S. Bastami et al., Semi-Inclusive Deep Inelastic Scattering in Wandzura-Wilczek-type approximation, JHEP 06 (2019) 007 [arXiv: 1807.10606] [INSPIRE].

[40] M. Gluck, E. Reya and A. Vogt, Pionic parton distributions, Z. Phys. C 53 (1992) 651 [INSPIRE].

[41] P.J. Sutton, A.D. Martin, R.G. Roberts and W.J. Stirling, Parton distributions for the pion extracted from Drell-Yan and prompt photon experiments, Phys. Rev. D 45 (1992) 2349 [INSPIRE].

[42] M. Gluck, E. Reya and I. Schienbein, Pionic parton distributions revisited, Eur. Phys. J. C 10 (1999) 313 [hep-ph/9903288] [INSPIRE].

[43] M. Aicher, A. Schafer and W. Vogelsang, Soft-gluon resummation and the valence parton distribution function of the pion, Phys. Rev. Lett. 105 (2010) 252003 [arXiv:1009.2481] [INSPIRE].

[44] P.C. Barry, N. Sato, W. Melnitchouk and C.-R. Ji, First Monte Carlo Global QCD Analysis of Pion Parton Distributions, Phys. Rev. Lett. 121 (2018) 152001 [arXiv:1804.01965] [INSPIRE].

[45] I. Novikov et al., Parton Distribution Functions of the Charged Pion Within The xFitter Framework, Phys. Rev. D 102 (2020) 014040 [arXiv:2002.02902] [INSPIRE].

[46] COMPASS collaboration, First measurement of transverse-spin-dependent azimuthal asymmetries in the Drell-Yan process, Phys. Rev. Lett. 119 (2017) 112002 [arXiv: 1704.00488] [INSPIRE].

[47] H. Weigel, E. Ruiz Arriola and L.P. Gamberg, Hadron structure functions in a chiral quark model: Regularization, scaling and sum rules, Nucl. Phys. B 560 (1999) 383 [hep-ph/9905329] [INSPIRE].

[48] E. Ruiz Arriola and W. Broniowski, Spectral quark model and low-energy hadron phenomenology, Phys. Rev. D 67 (2003) 074021 [hep-ph/0301202] [INSPIRE].

[49] R. Jakob, P.J. Mulders and J. Rodrigues, Modeling quark distribution and fragmentation functions, Nucl. Phys. A 626 (1997) 937 [hep-ph/9704335] [INSPIRE].

[50] L.P. Gamberg, G.R. Goldstein and M. Schlegel, Transverse Quark Spin Effects and the Flavor Dependence of the Boer-Mulders Function, Phys. Rev. D 77 (2008) 094016 [arXiv:0708.0324] [INSPIRE].

[51] L. Gamberg and M. Schlegel, Final state interactions and the transverse structure of the pion using non-perturbative eikonal methods, Phys. Lett. B 685 (2010) 95 [arXiv:0911.1964] [INSPIRE]. 
[52] A. Bacchetta, F. Conti and M. Radici, Transverse-momentum distributions in a diquark spectator model, Phys. Rev. D 78 (2008) 074010 [arXiv:0807.0323] [INSPIRE].

[53] Z. Lu and B.-Q. Ma, Non-zero transversity distribution of the pion in a quark-spectator-antiquark model, Phys. Rev. D 70 (2004) 094044 [hep-ph/0411043] [INSPIRE].

[54] B. Pasquini, S. Cazzaniga and S. Boffi, Transverse momentum dependent parton distributions in a light-cone quark model, Phys. Rev. D 78 (2008) 034025 [arXiv:0806.2298] [INSPIRE].

[55] B. Pasquini and F. Yuan, Sivers and Boer-Mulders functions in Light-Cone Quark Models, Phys. Rev. D 81 (2010) 114013 [arXiv:1001.5398] [InSPIRE].

[56] C. Lorce, B. Pasquini and M. Vanderhaeghen, Unified framework for generalized and transverse-momentum dependent parton distributions within a $3 Q$ light-cone picture of the nucleon, JHEP 05 (2011) 041 [arXiv: 1102.4704] [INSPIRE].

[57] S. Boffi, A.V. Efremov, B. Pasquini and P. Schweitzer, Azimuthal spin asymmetries in light-cone constituent quark models, Phys. Rev. D 79 (2009) 094012 [arXiv:0903.1271] [INSPIRE].

[58] B. Pasquini and P. Schweitzer, Naive time-reversal odd phenomena in semi-inclusive deep-inelastic scattering from light-cone constituent quark models, Phys. Rev. D $\mathbf{8 3}$ (2011) 114044 [arXiv: 1103.5977] [INSPIRE].

[59] B. Pasquini and P. Schweitzer, Pion transverse momentum dependent parton distributions in a light-front constituent approach, and the Boer-Mulders effect in the pion-induced Drell-Yan process, Phys. Rev. D 90 (2014) 014050 [arXiv:1406.2056] [INSPIRE].

[60] C. Lorcé, B. Pasquini and P. Schweitzer, Unpolarized transverse momentum dependent parton distribution functions beyond leading twist in quark models, JHEP 01 (2015) 103 [arXiv: 1411.2550] [INSPIRE].

[61] C. Lorcé, B. Pasquini and P. Schweitzer, Transverse pion structure beyond leading twist in constituent models, Eur. Phys. J. C 76 (2016) 415 [arXiv:1605.00815] [inSPIRE].

[62] F. Yuan, Sivers function in the MIT bag model, Phys. Lett. B 575 (2003) 45 [hep-ph/0308157] [INSPIRE].

[63] H. Avakian, A.V. Efremov, P. Schweitzer and F. Yuan, Transverse momentum dependent distribution function $h_{1 T}^{\perp}$ and the single spin asymmetry $A_{U T}^{\sin \left(3 \phi-\phi_{S}\right)}$, Phys. Rev. D 78 (2008) 114024 [arXiv:0805.3355] [INSPIRE].

[64] A. Courtoy, F. Fratini, S. Scopetta and V. Vento, A Quark model analysis of the Sivers function, Phys. Rev. D 78 (2008) 034002 [arXiv:0801.4347] [INSPIRE].

[65] A. Courtoy, S. Scopetta and V. Vento, Model calculations of the Sivers function satisfying the Burkardt Sum Rule, Phys. Rev. D 79 (2009) 074001 [arXiv:0811.1191] [InSPIRE].

[66] H. Avakian, A.V. Efremov, P. Schweitzer and F. Yuan, The transverse momentum dependent distribution functions in the bag model, Phys. Rev. D 81 (2010) 074035 [arXiv: 1001.5467 ] [INSPIRE].

[67] S. Noguera and S. Scopetta, Pion transverse momentum dependent parton distributions in the Nambu and Jona-Lasinio model, JHEP 11 (2015) 102 [arXiv:1508.01061] [INSPIRE]. 
[68] M. Engelhardt, P. Hägler, B. Musch, J. Negele and A. Schäfer, Lattice QCD study of the Boer-Mulders effect in a pion, Phys. Rev. D 93 (2016) 054501 [arXiv:1506.07826] [INSPIRE].

[69] M. Lambertsen and W. Vogelsang, Drell-Yan lepton angular distributions in perturbative QCD, Phys. Rev. D 93 (2016) 114013 [arXiv:1605.02625] [InSPIRE].

[70] W.-C. Chang, R.E. McClellan, J.-C. Peng and O. Teryaev, Lepton Angular Distributions of Fixed-target Drell-Yan Experiments in Perturbative QCD and a Geometric Approach, Phys. Rev. D 99 (2019) 014032 [arXiv:1811.03256] [INSPIRE].

[71] A. Bacchetta, S. Cotogno and B. Pasquini, The transverse structure of the pion in momentum space inspired by the AdS/QCD correspondence, Phys. Lett. B 771 (2017) 546 [arXiv: 1703. 07669] [INSPIRE].

[72] W. Broniowski and E. Ruiz Arriola, Partonic quasidistributions of the proton and pion from transverse-momentum distributions, Phys. Rev. D 97 (2018) 034031 [arXiv:1711.03377] [INSPIRE].

[73] F.A. Ceccopieri, A. Courtoy, S. Noguera and S. Scopetta, Pion nucleus Drell-Yan process and parton transverse momentum in the pion, Eur. Phys. J. C 78 (2018) 644 [arXiv: 1801.07682] [INSPIRE].

[74] M. Traini, A. Mair, A. Zambarda and V. Vento, Constituent quarks and parton distributions, Nucl. Phys. A 614 (1997) 472 [INSPIRE].

[75] T. Gehrmann, T. Luebbert and L.L. Yang, Calculation of the transverse parton distribution functions at next-to-next-to-leading order, JHEP 06 (2014) 155 [arXiv:1403.6451] [INSPIRE].

[76] M.G. Echevarria, I. Scimemi and A. Vladimirov, Universal transverse momentum dependent soft function at NNLO, Phys. Rev. D 93 (2016) 054004 [arXiv:1511.05590] [INSPIRE].

[77] M.G. Echevarria, I. Scimemi and A. Vladimirov, Transverse momentum dependent fragmentation function at next-to-next-to-leading order, Phys. Rev. D 93 (2016) 011502 [Erratum ibid. 94 (2016) 099904] [arXiv:1509.06392] [INSPIRE].

[78] M.G. Echevarria, I. Scimemi and A. Vladimirov, Unpolarized Transverse Momentum Dependent Parton Distribution and Fragmentation Functions at next-to-next-to-leading order, JHEP 09 (2016) 004 [arXiv: 1604.07869] [INSPIRE].

[79] Y. Li and H.X. Zhu, Bootstrapping Rapidity Anomalous Dimensions for Transverse-Momentum Resummation, Phys. Rev. Lett. 118 (2017) 022004 [arXiv: 1604.01404] [INSPIRE].

[80] A.A. Vladimirov, Correspondence between Soft and Rapidity Anomalous Dimensions, Phys. Rev. Lett. 118 (2017) 062001 [arXiv:1610.05791] [INSPIRE].

[81] M.-X. Luo, X. Wang, X. Xu, L.L. Yang, T.-Z. Yang and H.X. Zhu, Transverse Parton Distribution and Fragmentation Functions at NNLO: the Quark Case, JHEP 10 (2019) 083 [arXiv: 1908.03831] [INSPIRE].

[82] M.-x. Luo, T.-Z. Yang, H.X. Zhu and Y.J. Zhu, Quark Transverse Parton Distribution at the Next-to-Next-to-Next-to-Leading Order, Phys. Rev. Lett. 124 (2020) 092001 [arXiv: 1912.05778] [INSPIRE].

[83] M.A. Ebert, B. Mistlberger and G. Vita, Transverse momentum dependent PDFs at $N^{3} L O$, JHEP 09 (2020) 146 [arXiv: 2006.05329] [INSPIRE]. 
[84] D. Gutiérrez-Reyes, I. Scimemi and A.A. Vladimirov, Twist-2 matching of transverse momentum dependent distributions, Phys. Lett. B 769 (2017) 84 [arXiv:1702.06558] [INSPIRE].

[85] D. Gutierrez-Reyes, I. Scimemi and A. Vladimirov, Transverse momentum dependent transversely polarized distributions at next-to-next-to-leading-order, JHEP 07 (2018) 172 [arXiv: 1805.07243] [INSPIRE].

[86] X. Ji, J.-W. Qiu, W. Vogelsang and F. Yuan, A Unified picture for single transverse-spin asymmetries in hard processes, Phys. Rev. Lett. 97 (2006) 082002 [hep-ph/0602239] [INSPIRE].

[87] Y. Koike, W. Vogelsang and F. Yuan, On the Relation Between Mechanisms for Single-Transverse-Spin Asymmetries, Phys. Lett. B 659 (2008) 878 [arXiv:0711.0636] [INSPIRE].

[88] P. Sun and F. Yuan, Transverse momentum dependent evolution: Matching semi-inclusive deep inelastic scattering processes to Drell-Yan and $W / Z$ boson production, Phys. Rev. $D$ 88 (2013) 114012 [arXiv:1308.5003] [INSPIRE].

[89] L.-Y. Dai, Z.-B. Kang, A. Prokudin and I. Vitev, Next-to-leading order transverse momentum-weighted Sivers asymmetry in semi-inclusive deep inelastic scattering: the role of the three-gluon correlator, Phys. Rev. D 92 (2015) 114024 [arXiv:1409.5851] [InSPIRE].

[90] I. Scimemi, A. Tarasov and A. Vladimirov, Collinear matching for Sivers function at next-to-leading order, JHEP 05 (2019) 125 [arXiv: 1901.04519] [INSPIRE].

[91] V. Moos and A. Vladimirov, Calculation of transverse momentum dependent distributions beyond the leading power, JHEP 12 (2020) 145 [arXiv: 2008.01744] [INSPIRE].

[92] P. Schweitzer, T. Teckentrup and A. Metz, Intrinsic transverse parton momenta in deeply inelastic reactions, Phys. Rev. D 81 (2010) 094019 [arXiv:1003.2190] [INSPIRE].

[93] P. Sun, J. Isaacson, C.P. Yuan and F. Yuan, Nonperturbative functions for SIDIS and Drell-Yan processes, Int. J. Mod. Phys. A 33 (2018) 1841006 [arXiv:1406.3073] [inSPIRE].

[94] Z.-B. Kang, A. Prokudin, P. Sun and F. Yuan, Nucleon tensor charge from Collins azimuthal asymmetry measurements, Phys. Rev. D 91 (2015) 071501 [arXiv:1410.4877] [INSPIRE].

[95] Z.-B. Kang, A. Prokudin, P. Sun and F. Yuan, Extraction of Quark Transversity Distribution and Collins Fragmentation Functions with QCD Evolution, Phys. Rev. D 93 (2016) 014009 [arXiv: 1505.05589] [inSPIRE].

[96] A. Bacchetta, F. Delcarro, C. Pisano, M. Radici and A. Signori, Extraction of partonic transverse momentum distributions from semi-inclusive deep-inelastic scattering, Drell-Yan and Z-boson production, JHEP 06 (2017) 081 [Erratum ibid. 06 (2019) 051] [arXiv:1703.10157] [INSPIRE].

[97] X. Wang, Z. Lu and I. Schmidt, Transverse momentum spectrum of dilepton pair in the unpolarized $\pi^{-} N$ Drell-Yan process within TMD factorization, JHEP 08 (2017) 137 [arXiv: 1707.05207] [INSPIRE].

[98] A. Vladimirov, Pion-induced Drell-Yan processes within TMD factorization, JHEP 10 (2019) 090 [arXiv: 1907.10356] [InSPIRE]. 
[99] H. Li, X. Wang and Z. Lu, $\sin \left(2 \phi-\phi_{S}\right)$ azimuthal asymmetry in the pion induced Drell-Yan process within TMD factorization, Phys. Rev. D 101 (2020) 054013 [arXiv:1907.07095] [INSPIRE].

[100] X. Wang, W. Mao and Z. Lu, Boer-Mulders effect in the unpolarized pion induced Drell-Yan process at COMPASS within TMD factorization, Eur. Phys. J. C 78 (2018) 643 [arXiv:1805.03017] [INSPIRE].

[101] X. Wang and Z. Lu, Sivers asymmetry in the pion induced Drell-Yan process at COMPASS within transverse momentum dependent factorization, Phys. Rev. D 97 (2018) 054005 [arXiv: 1801.00660] [INSPIRE].

[102] P. Ratcliffe, Radiative Corrections to the Helicity Asymmetries for the Drell-Yan Process in QCD, Nucl. Phys. B 223 (1983) 45 [INSPIRE].

[103] A. Weber, Soft gluon resummations for polarized Drell-Yan dimuon production, Nucl. Phys. B 382 (1992) 63 [INSPIRE].

[104] W. Vogelsang and A. Weber, Drell-Yan dimuon production with transversely polarized protons, Phys. Rev. D 48 (1993) 2073 [InSPIRE].

[105] A.P. Contogouris, B. Kamal and Z. Merebashvili, One loop corrections to lepton pair production by transversely polarized hadrons, Phys. Lett. B 337 (1994) 169 [INSPIRE].

[106] T. Gehrmann, QCD corrections to the longitudinally polarized Drell-Yan process, Nucl. Phys. B 498 (1997) 245 [hep-ph/9702263] [INSPIRE].

[107] G. Bunce, N. Saito, J. Soffer and W. Vogelsang, Prospects for spin physics at RHIC, Ann. Rev. Nucl. Part. Sci. 50 (2000) 525 [hep-ph/0007218] [inSPIRE].

[108] H. Shimizu, G.F. Sterman, W. Vogelsang and H. Yokoya, Dilepton production near partonic threshold in transversely polarized proton-antiproton collisions, Phys. Rev. D 71 (2005) 114007 [hep-ph/0503270] [INSPIRE].

[109] I. Scimemi and A. Vladimirov, Systematic analysis of double-scale evolution, JHEP 08 (2018) 003 [arXiv: 1803.11089] [INSPIRE].

[110] J.C. Collins and D.E. Soper, Back-To-Back Jets: Fourier Transform from B to K-Transverse, Nucl. Phys. B 197 (1982) 446 [inSPIRE].

[111] R. Angeles-Martinez et al., Transverse Momentum Dependent (TMD) parton distribution functions: status and prospects, Acta Phys. Polon. B 46 (2015) 2501 [arXiv:1507.05267] [INSPIRE].

[112] M.A. Ebert, J.K.L. Michel, I.W. Stewart and F.J. Tackmann, Drell-Yan $q_{T}$ Resummation of Fiducial Power Corrections at $N^{3} L L$, arXiv:2006.11382 [INSPIRE].

[113] J. Collins and T.C. Rogers, Connecting Different TMD Factorization Formalisms in QCD, Phys. Rev. D 96 (2017) 054011 [arXiv: 1705. 07167] [InSPIRE].

[114] U. D'Alesio and F. Murgia, Parton intrinsic motion in inclusive particle production: Unpolarized cross sections, single spin asymmetries and the Sivers effect, Phys. Rev. D 70 (2004) 074009 [hep-ph/0408092] [INSPIRE].

[115] A.V. Efremov, P. Schweitzer, O.V. Teryaev and P. Zavada, The relation between TMDs and PDFs in the covariant parton model approach, Phys. Rev. D 83 (2011) 054025

[arXiv: 1012.5296] [INSPIRE]. 
[116] P. Schweitzer, M. Strikman and C. Weiss, Intrinsic transverse momentum and parton correlations from dynamical chiral symmetry breaking, JHEP 01 (2013) 163 [arXiv:1210.1267] [INSPIRE].

[117] D. Boer, L. Gamberg, B. Musch and A. Prokudin, Bessel-Weighted Asymmetries in Semi Inclusive Deep Inelastic Scattering, JHEP 10 (2011) 021 [arXiv:1107.5294] [INSPIRE].

[118] A. Bacchetta, G. Bozzi, M.G. Echevarria, C. Pisano, A. Prokudin and M. Radici, Azimuthal asymmetries in unpolarized SIDIS and Drell-Yan processes: a case study towards TMD factorization at subleading twist, Phys. Lett. B 797 (2019) 134850 [arXiv:1906.07037].

[119] J.-w. Qiu and X.-f. Zhang, QCD prediction for heavy boson transverse momentum distributions, Phys. Rev. Lett. 86 (2001) 2724 [hep-ph/0012058] [INSPIRE].

[120] S. Moch, J.A.M. Vermaseren and A. Vogt, The Quark form-factor at higher orders, JHEP 08 (2005) 049 [hep-ph/0507039] [INSPIRE].

[121] Z.-B. Kang, B.-W. Xiao and F. Yuan, QCD Resummation for Single Spin Asymmetries, Phys. Rev. Lett. 107 (2011) 152002 [arXiv:1106.0266] [INSPIRE].

[122] M.G. Echevarria, A. Idilbi, A. Schäfer and I. Scimemi, Model-Independent Evolution of Transverse Momentum Dependent Distribution Functions (TMDs) at NNLL, Eur. Phys. J. C 73 (2013) 2636 [arXiv:1208.1281] [INSPIRE].

[123] A. Grozin, J.M. Henn, G.P. Korchemsky and P. Marquard, Three Loop Cusp Anomalous Dimension in QCD, Phys. Rev. Lett. 114 (2015) 062006 [arXiv: 1409.0023] [INSPIRE].

[124] M.G. Echevarria, Z.-B. Kang and J. Terry, Global analysis of the Sivers functions at $N L O+N N L L$ in $Q C D$, JHEP 01 (2021) 126 [arXiv: 2009.10710] [INSPIRE].

[125] S. Bethke, World Summary of $\alpha_{s}$ (2012), Nucl. Phys. B Proc. Suppl. 234 (2013) 229 [arXiv:1210.0325] [INSPIRE].

[126] T.-J. Hou et al., New CTEQ global analysis of quantum chromodynamics with high-precision data from the LHC, Phys. Rev. D 103 (2021) 014013 [arXiv:1912.10053] [INSPIRE].

[127] M. Anselmino, M. Boglione, U. D’Alesio, S. Melis, F. Murgia and A. Prokudin, New insight on the Sivers transverse momentum dependent distribution function, J. Phys. Conf. Ser. 295 (2011) 012062 [arXiv: 1012.3565] [inSPIRE].

[128] M. Anselmino, M. Boglione, U. D'Alesio, A. Kotzinian, F. Murgia and A. Prokudin, Extracting the Sivers function from polarized SIDIS data and making predictions, Phys. Rev. D 72 (2005) 094007 [Erratum ibid. 72 (2005) 099903] [hep-ph/0507181] [INSPIRE].

[129] M. Anselmino et al., Comparing extractions of Sivers functions, in International Workshop on Transverse Polarization Phenomena in Hard Processes, Como Italy (2005), pg. 236 [hep-ph/0511017] [INSPIRE].

[130] J.C. Collins, A.V. Efremov, K. Goeke, S. Menzel, A. Metz and P. Schweitzer, Sivers effect in semi-inclusive deeply inelastic scattering, Phys. Rev. D 73 (2006) 014021 [hep-ph/0509076] [INSPIRE].

[131] W. Vogelsang and F. Yuan, Single-transverse spin asymmetries: From DIS to hadronic collisions, Phys. Rev. D 72 (2005) 054028 [hep-ph/0507266] [INSPIRE].

[132] M. Anselmino et al., Sivers Effect for Pion and Kaon Production in Semi-Inclusive Deep Inelastic Scattering, Eur. Phys. J. A 39 (2009) 89 [arXiv:0805.2677] [INSPIRE]. 
[133] A. Bacchetta and M. Radici, Constraining quark angular momentum through semi-inclusive measurements, Phys. Rev. Lett. 107 (2011) 212001 [arXiv:1107.5755] [INSPIRE].

[134] M.G. Echevarria, A. Idilbi, Z.-B. Kang and I. Vitev, QCD Evolution of the Sivers Asymmetry, Phys. Rev. D 89 (2014) 074013 [arXiv:1401.5078] [INSPIRE].

[135] A. Bacchetta, F. Delcarro, C. Pisano and M. Radici, The three-dimensional distribution of quarks in momentum space, arXiv:2004.14278 [INSPIRE].

[136] J.C. Collins, Fragmentation of transversely polarized quarks probed in transverse momentum distributions, Nucl. Phys. B 396 (1993) 161 [hep-ph/9208213] [INSPIRE].

[137] HERMES collaboration, Observation of a single spin azimuthal asymmetry in semiinclusive pion electro production, Phys. Rev. Lett. 84 (2000) 4047 [hep-ex/9910062] [INSPIRE].

[138] CLAS collaboration, Semi-Inclusive $\pi_{0}$ target and beam-target asymmetries from $6 \mathrm{GeV}$ electron scattering with CLAS, Phys. Lett. B 782 (2018) 662 [arXiv:1709.10054] [INSPIRE].

[139] B. Parsamyan, Measurement of target-polarization dependent azimuthal asymmetries in SIDIS and Drell-Yan processes at COMPASS experiment, PoS (QCDEV2017) 042 [INSPIRE].

[140] F. Schlumpf, Nucleon form-factors in a relativistic quark model, J. Phys. G 20 (1994) 237 [hep-ph/9301233] [INSPIRE].

[141] B. Pasquini and S. Boffi, Electroweak structure of the nucleon, meson cloud and light-cone wavefunctions, Phys. Rev. D 76 (2007) 074011 [arXiv:0707.2897] [InSPIRE].

[142] F. Schlumpf, Charge form-factors of pseudoscalar mesons, Phys. Rev. D 50 (1994) 6895 [hep-ph/9406267] [INSPIRE].

[143] L.P. Gamberg and G.R. Goldstein, T-odd effects in unpolarized Drell-Yan scattering, Phys. Lett. B 650 (2007) 362 [hep-ph/0506127] [INSPIRE].

[144] H. Meyer and P.J. Mulders, Polarized and unpolarized structure functions in a diquark model for the nucleon, Nucl. Phys. A 528 (1991) 589 [INSPIRE].

[145] S.J. Brodsky, D.S. Hwang and I. Schmidt, Final state interactions and single spin asymmetries in semiinclusive deep inelastic scattering, Phys. Lett. B $5 \mathbf{5 0}$ (2002) 99 [hep-ph/0201296] [INSPIRE].

[146] X.-d. Ji and F. Yuan, Parton distributions in light cone gauge: Where are the final state interactions?, Phys. Lett. B $\mathbf{5 4 3}$ (2002) 66 [hep-ph/0206057] [INSPIRE].

[147] G.R. Goldstein and L. Gamberg, Transversity and meson photoproduction, in 31st International Conference on High Energy Physics, Amsterdam The Netherlands (2002), pg. 452 [hep-ph/0209085] [INSPIRE].

[148] A. Metz, Gluon-exchange in spin-dependent fragmentation, Phys. Lett. B 549 (2002) 139 [hep-ph/0209054] [INSPIRE].

[149] L.P. Gamberg, G.R. Goldstein and K.A. Oganessyan, A Mechanism for the T odd pion fragmentation function, Phys. Rev. D 68 (2003) 051501 [hep-ph/0307139] [INSPIRE].

[150] J.C. Collins and A. Metz, Universality of soft and collinear factors in hard-scattering factorization, Phys. Rev. Lett. 93 (2004) 252001 [hep-ph/0408249] [INSPIRE].

[151] L.P. Gamberg, A. Mukherjee and P.J. Mulders, Spectral analysis of gluonic pole matrix elements for fragmentation, Phys. Rev. D 77 (2008) 114026 [arXiv:0803.2632] [INSPIRE]. 
[152] L.P. Gamberg, G.R. Goldstein and K.A. Oganessyan, Novel transversity properties in semiinclusive deep inelastic scattering, Phys. Rev. D 67 (2003) 071504 [hep-ph/0301018] [INSPIRE].

[153] D. Boer, S.J. Brodsky and D.S. Hwang, Initial state interactions in the unpolarized Drell-Yan process, Phys. Rev. D 67 (2003) 054003 [hep-ph/0211110] [InSPIRE].

[154] M. Hirai, S. Kumano and M. Miyama, Numerical solution of $Q^{2}$ evolution equation for the transversity distribution Delta(T)q, Comput. Phys. Commun. 111 (1998) 150 [hep-ph/9712410] [INSPIRE].

[155] COMPASS collaboration, Transversely polarized Drell-Yan measurements at COMPASS, PoS (DIS2019) 195 [arXiv: 1908.01727] [INSPIRE].

[156] J.C. Collins, Leading twist single transverse-spin asymmetries: Drell-Yan and deep inelastic scattering, Phys. Lett. B 536 (2002) 43 [hep-ph/0204004] [InSPIRE].

[157] S.J. Brodsky, D.S. Hwang and I. Schmidt, Initial state interactions and single spin asymmetries in Drell-Yan processes, Nucl. Phys. B 642 (2002) 344 [hep-ph/0206259] [INSPIRE].

[158] E.-C. Aschenauer et al., The RHIC SPIN Program: Achievements and Future Opportunities, arXiv:1501.01220 [INSPIRE].

[159] STAR collaboration, Measurement of the transverse single-spin asymmetry in $p^{\uparrow}+p \rightarrow W^{ \pm} / Z^{0}$ at RHIC, Phys. Rev. Lett. 116 (2016) 132301 [arXiv:1511.06003] [INSPIRE].

[160] M. Anselmino, M. Boglione, U. D'Alesio, F. Murgia and A. Prokudin, Study of the sign change of the Sivers function from STAR Collaboration $W / Z$ production data, JHEP 04 (2017) 046 [arXiv:1612.06413] [INSPIRE].

[161] A.V. Efremov, K. Goeke, S. Menzel, A. Metz and P. Schweitzer, Sivers effect in semi-inclusive DIS and in the Drell-Yan process, Phys. Lett. B 612 (2005) 233 [hep-ph/0412353] [INSPIRE].

[162] J.C. Collins et al., Sivers effect in Drell-Yan at RHIC, Phys. Rev. D 73 (2006) 094023 [hep-ph/0511272] [INSPIRE].

[163] M. Anselmino, M. Boglione, U. D’Alesio, S. Melis, F. Murgia and A. Prokudin, Sivers effect in Drell-Yan processes, Phys. Rev. D 79 (2009) 054010 [arXiv:0901.3078] [INSPIRE].

[164] D. Boer, Investigating the origins of transverse spin asymmetries at RHIC, Phys. Rev. D 60 (1999) 014012 [hep-ph/9902255] [INSPIRE].

[165] NA10 collaboration, Angular Distributions of Muon Pairs Produced by Negative Pions on Deuterium and Tungsten, Z. Phys. C 37 (1988) 545 [inSPIRE].

[166] J.S. Conway et al., Experimental Study of Muon Pairs Produced by 252-GeV Pions on Tungsten, Phys. Rev. D 39 (1989) 92 [inSPIRE].

[167] V. Barone, S. Melis and A. Prokudin, Azimuthal asymmetries in unpolarized Drell-Yan processes and the Boer-Mulders distributions of antiquarks, Phys. Rev. D 82 (2010) 114025 [arXiv: 1009.3423] [INSPIRE].

[168] COMPASS collaboration, COMPASS-II Proposal, SPSC-P-340, CERN-SPSC-2010-014 (2010). 
[169] NuSea collaboration, Measurement of Angular Distributions of Drell-Yan Dimuons in $p+d$ Interaction at 800-GeV/c, Phys. Rev. Lett. 99 (2007) 082301 [hep-ex/0609005] [InSPIRE].

[170] NuSEa collaboration, Measurement of Angular Distributions of Drell-Yan Dimuons in $p+p$ Interactions at 800-GeV/c, Phys. Rev. Lett. 102 (2009) 182001 [arXiv:0811.4589] [INSPIRE].

[171] M. Burkardt and B. Hannafious, Are all Boer-Mulders functions alike?, Phys. Lett. B 658 (2008) 130 [arXiv:0705.1573] [InSPIRE].

[172] P.V. Pobylitsa, Transverse momentum dependent parton distributions in large $N_{c} Q C D$, hep-ph/0301236 [INSPIRE].

[173] A. Sissakian, O. Shevchenko, A. Nagaytsev, O. Denisov and O. Ivanov, Transversity and its accompanying T-odd distribution from Drell-Yan processes with pion-proton collisions, Eur. Phys. J. C 46 (2006) 147 [hep-ph/0512095] [INSPIRE].

[174] I.A. Savin et al., Spin Physics Experiments at NICA-SPD with polarized proton and deuteron beams, EPJ Web Conf. 85 (2015) 02039 [arXiv:1408.3959] [INSPIRE]. 